\title{
Robinson-Schensted correspondence for the signed Brauer algebras
}

\author{
M. Parvathi and A. Tamilselvi \\ Ramanujan Institute for Advanced Study in Mathematics \\ University of Madras, Chennai-600 005, India \\ sparvathi.riasm@gmail.com, tamilselvi.riasm@gmail.com
}

Submitted: Jan 31, 2007; Accepted: Jul 5, 2007; Published: Jul 19, 2007

Mathematics Subject Classifications: 05E10, 20C30

\begin{abstract}
In this paper, we develop the Robinson-Schensted correspondence for the signed Brauer algebra. The Robinson-Schensted correspondence gives the bijection between the set of signed Brauer diagrams $d$ and the pairs of standard bi-domino tableaux of shape $\lambda=\left(\lambda_{1}, \lambda_{2}\right)$ with $\lambda_{1}=\left(2^{2 f}\right), \lambda_{2} \in \bar{\Gamma}_{f, r}$ where $\bar{\Gamma}_{f, r}=\{\lambda \mid \lambda \vdash$ $2(n-2 f)+\left|\delta_{r}\right|$ whose 2 -core is $\left.\delta_{\mathrm{r}}, \delta_{r}=(r, r-1, \ldots, 1,0)\right\}$, for fixed $r \geq 0$ and $0 \leq f \leq\left[\frac{n}{2}\right]$. We also give the Robinson-Schensted for the signed Brauer algebra using the vacillating tableau which gives the bijection between the set of signed Brauer diagrams $\bar{V}_{n}$ and the pairs of $d$-vacillating tableaux of shape $\lambda \in \bar{\Gamma}_{f, r}$ and $0 \leq f \leq\left[\frac{n}{2}\right]$. We derive the Knuth relations and the determinantal formula for the signed Brauer algebra by using the Robinson-Schensted correspondence for the standard bi-dominotableau whose core is $\delta_{r}, r \geq n-1$.
\end{abstract}

\section{Introduction}

In $[\mathrm{PK}]$, it has been observed that the number of signed Brauer diagrams is the dimension of the regular representation of the signed Brauer algebra, whereas by Artin-Wedderburn structure theorem, the dimension of the regular representation is the sum of the squares of the dimension of the irreducible representations of the signed Brauer algebra which are indexed by partitions $\lambda \in \bar{\Gamma}_{f, r}$ where $\bar{\Gamma}_{f, r}=\left\{\lambda|\lambda \vdash 2(n-2 f)+| \delta_{r} \mid\right.$ whose 2 -core is $\delta_{\mathrm{r}}, \delta_{\mathrm{r}}=$ $(\mathrm{r}, \mathrm{r}-1, \ldots, 1,0)\}$, for fixed $r \geq 0$ and $0 \leq f \leq\left[\frac{n}{2}\right]$.

This motivated us to construct an explicit bijection between the set of signed Brauer diagrams $\bar{V}_{n}$ and the pairs of $d$-vacillating tableaux of shape $\lambda \in \bar{\Gamma}_{f, r}$, for fixed $r \geq 0$ and

$0 \leq f \leq\left[\frac{n}{2}\right]$. We also construct the Robinson-Schensted correspondence for the signed Brauer algebra which gives the bijection between the set of signed Brauer diagrams $d$ and the pairs of standard bi-dominotableaux of shape $\lambda=\left(\lambda_{1}, \lambda_{2}\right)$ with $\lambda_{1}=\left(2^{2 f}\right), \lambda_{2} \in \bar{\Gamma}_{f, r}$, for fixed $r \geq 0$ and $0 \leq f \leq\left[\frac{n}{2}\right]$, which are generalisation of bitableaux introduced by 
Enyang [E], while constructing the cell modules for the Birman-Murakami-Wenzl algebras and Brauer algebras with bases indexed by certain bitableau. We also give the method for translating the vacillating tableau to the bi-domino tableau.

We also give the Knuth relations and the determinantal formula for the signed Brauer algebra. Since the Brauer algebra is the subalgebra of the signed Brauer algebra, our correspondence restricted to the Brauer algebra is the same as in [DS, HL, Ro1, Ro2, Su]. As a biproduct, we give the Knuth relations and the determinantal formula for the Brauer algebra.

\section{Preliminaries}

We state the basic definitions and some known results which will be used in this paper.

Definition 2.1. [S] A sequence of non-negative integers $\lambda=\left(\lambda_{1}, \lambda_{2}, \ldots\right)$ is called a partition of $n$, which is denoted by $\lambda \vdash n$, if

1. $\lambda_{i} \geq \lambda_{i+1}$, for every $i \geq 1$

2. $\sum_{i=1}^{\infty} \lambda_{i}=n$

The $\lambda_{i}$ are called the parts of $\lambda$.

Definition 2.2. [S] Suppose $\lambda=\left(\lambda_{1}, \lambda_{2}, \ldots, \lambda_{l}\right) \vdash n$. The Young diagram of $\lambda$ is an array of $n$ dots having $l$ left justified rows with row $i$ containing $\lambda_{i}$ dots for $1 \leq i \leq l$.

Example 2.3.

$$
\begin{array}{ccccccc}
* & * & \cdot & \cdot & \cdot & * & \lambda_{1} \text { nodes } \\
* & * & \cdot & \cdot & * & \lambda_{2} \text { nodes } \\
\cdot \lambda]:= & \cdot & & & & \cdot \\
\cdot & \cdot & & & & \cdot \\
\cdot & \cdot & & & & \cdot \\
& * & * & \cdots & * & & \lambda_{r} \text { nodes }
\end{array}
$$

Definition 2.4. [JK] Let $\alpha$ be a partition of $n$, denoted by $\alpha \vdash n$. Then the $(i, j)$-hook of $\alpha$, denoted by $H_{i, j}^{\alpha}$ which is defined to be a $\Gamma$-shaped subset of diagram $\alpha$ which consists of the $(i, j)$-node called the corner of the hook and all the nodes to the right of it in the same row together with all the nodes lower down and in the same column as the corner.

The number $h_{i j}$ of nodes of $H_{i j}^{\alpha}$ i.e.,

$$
h_{i j}=\alpha_{i}-j+\alpha_{j}^{\prime}-i+1
$$

where $\alpha_{j}^{\prime}=$ number of nodes in the $j$ th column of $\alpha$, is called the length of $H_{i, j}^{\alpha}$, where $\alpha=\left[\alpha_{1}, \cdots \alpha_{k}\right]$. A hook of length $q$ is called a $q$-hook. Then $H[\alpha]=\left(h_{i j}\right)$ is called the hook graph of $\alpha$. 
Definition 2.5. [R] We shall call the $(i, j)$ node of $\lambda$, an $r$-node if and only if $j-i \equiv$ $r(\bmod 2)$.

Definition 2.6. [R] A node $(i, j)$ is said to be a $(2, r)$ node if $h_{i j}=2 m$ and the residue of node $\left(i, \lambda_{i}\right)$ in $\lambda$ is $r$. i.e. $\lambda_{i}-i \equiv r(\bmod 2)$.

Definition 2.7. [R]. If we delete all the elements in the hook graph $H[\lambda]$ not divisible by 2 , then the remaining elements,

$$
h_{i j}=h_{i j}^{r}(2),(r=0,1)
$$

can be divided into disjoint sets whose $(2, r)$ nodes constitute the diagram $[\lambda]_{2}^{r},(r=0,1)$ with hook graph $\left(h_{i j}^{r}\right)$. The $\lambda$ is written as $\left(\lambda_{1}, \lambda_{2}\right)$ where the nodes in $\lambda_{1}$ correspond to $(2,0)$ nodes and the nodes in $\lambda_{2}$ correspond to $(2,1)$ nodes.

Definition 2.8. [JK] Let $\lambda \vdash n$. An $(i, j)$-node of $\lambda$ is said to be a rim node if there does not exist any $(i+1, j+1)$-node of $\lambda$.

Definition 2.9. [JK] A 2-hook comprising of rim nodes is called a rim 2-hook.

Definition 2.10. [JK] A Young diagram $\lambda$ which does not contain any 2-hook is called 2-core.

Definition 2.11. [JK] Each $2 \times 1$ and $1 \times 2$ rectangular boxes consisting of two nodes is called as a domino.

Lemma 2.12. [PST] Let $\rho \in \bar{\Gamma}_{0, r}$, for fixed $r \geq 0$. Then $\rho$ can be associated to a pair of partitions as in Definition 2.7, but when associated to a pair of partitions through the map $\eta$ we have, every domino in row $i$ of $\rho$ corresponds to a node of $\lambda_{i}$ and every domino in column $j$ of $\rho$ corresponds to a node of $\mu_{j}^{\prime}$.

Proposition 2.13. [PST] If $x \in \widetilde{S}_{n}$, the hyperoctahedral group of type $B_{n}$ then $P\left(x^{-1}\right)=$ $Q(x)$ and $Q\left(x^{-1}\right)=P(x)$ where $P(x), Q(x), P\left(x^{-1}\right), Q\left(x^{-1}\right)$ are the standard tableaux of shape $\lambda \in \bar{\Gamma}_{0, r}$, for fixed $r \geq n-1$.

Proposition 2.14. [BI, PST] If $x, y \in \widetilde{S}_{n}$, the hyperoctahedral group of type $B_{n}$ then $x \stackrel{K}{\sim} y \Longleftrightarrow P(x)=P(y)$ where $P(x), P(y)$ are the standard tableaux of shape $\lambda \in \bar{\Gamma}_{0, r}$, for fixed $r \geq n-1$.

Definition 2.15. [PST] Let $\rho \in \bar{\Gamma}_{0, r}, r \geq 0$. We define a map $\eta: \rho \mapsto\left(\rho^{(1)}, \rho^{(2)}\right), \lambda \vdash$ $l, \mu \vdash m, l+m=n$ such that if $r$ is even

$$
\begin{aligned}
& \rho_{i}^{(1)}=\frac{1}{2}\left(\rho_{i}-(n-i)\right) \text { if } \rho_{i}>n-i \\
& \rho_{i}^{(2)}=\sum_{\substack{j \\
\mu_{j}^{\prime} \geq i}} 1 \text { where } \rho_{\mathrm{j}}^{(2)^{\prime}}=\frac{1}{2}\left(\rho_{\mathrm{j}}^{\prime}-(\mathrm{n}-\mathrm{j})\right) \text { if } \rho_{\mathrm{j}}^{\prime}>\mathrm{n}-\mathrm{j}
\end{aligned}
$$


if $r$ is odd

$$
\begin{aligned}
\rho_{i}^{(1)} & =\frac{1}{2}\left(\rho_{i}-(n-i)+1\right) \text { if } \rho_{i}>n-i-1 \\
\rho_{i}^{(2)} & =\sum_{\substack{j \\
\mu_{j}^{\prime} \geq i}} 1 \quad \text { where } \quad \rho_{\mathrm{j}}^{\prime(2)}=\frac{1}{2}\left(\rho_{\mathrm{j}}^{\prime}-(\mathrm{n}-\mathrm{j})+1\right) \text { if } \rho_{\mathrm{j}}^{\prime}>\mathrm{n}-\mathrm{j}-1
\end{aligned}
$$

Proposition 2.16. [S] If $\lambda=\left(\lambda_{1}, \lambda_{2}, \ldots, \lambda_{l}\right) \vdash n$ then $f^{\lambda}=n !\left|\frac{1}{\left(\lambda_{i}-i+j\right) !}\right|_{l \times l}$.

\subsection{The Brauer algebras}

Definition 2.17. [Br] A Brauer graph is a graph on $2 n$ vertices with $n$ edges, vertices being arranged in two rows each row consisting of $n$ vertices and every vertex is the vertex of only one edge.

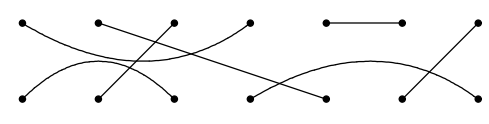

Definition 2.18. $[\mathrm{Br}]$ Let $V_{n}$ denote the set of Brauer graphs on $2 n$ vertices. Let $d, d^{\prime} \in$ $V_{n}$. The multiplication of two graphs is defined as follows:

1. Place $d$ above $d^{\prime}$.

2. join the $i^{\text {th }}$ lower vertex of $d$ with $i^{\text {th }}$ upper vertex of $d^{\prime}$

3. Let $c$ be the resulting graph obtain without loops. Then $a b=x^{r} c$, where $r$ is the number of loops, and $x$ is a variable.

For example,

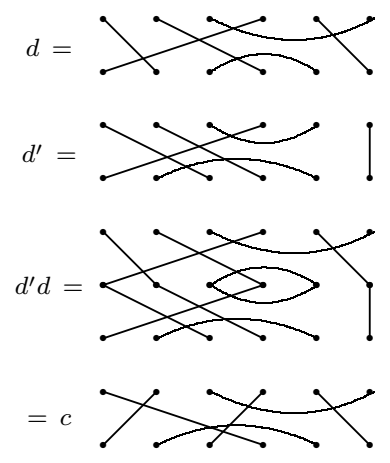

The Brauer algebra $D_{n}(x)$, where $x$ is an indeterminate, is the span of the diagrams on $n$ dots where the multiplication for the basis elements defined above. The dimension of $D_{n}(x)$ is $(2 n) ! !=(2 n-1)(2 n-3) \ldots 3.1$. 


\subsection{The signed Brauer algebras}

Definition 2.19. [PK] A signed diagram is a Brauer graph in which every edge is labeled by $\mathrm{a}+$ or a $-\operatorname{sign}$.

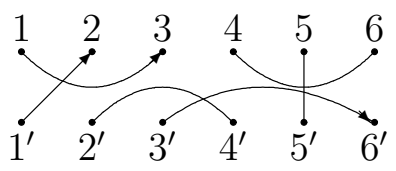

Definition 2.20. $[\mathrm{PK}]$ Let $\overline{V_{n}}$ denote the set of all signed Brauer graphs on $2 n$ vertices with $n$ signed edges.

Let $\bar{D}_{n}(x)$ denote the linear span of $\overline{V_{n}}$ where $x$ is an indeterminate. The dimension of $\bar{D}_{n}(x)$ is $2^{n}(2 n) ! !=2^{n}(2 n-1)(2 n-3) \ldots 3.1$.

Let $\bar{a}, \bar{b} \in \bar{V}_{n}$. Since $a, b$ are Brauer graphs, $a b=x^{d} c$, the only thing we have to do is to assign a direction for every edge. An edge $\alpha$ in the product $\bar{a} \bar{b}$ will be labeled as a + or a - sign according as the number of negative edges involved from $\bar{a}$ and $\bar{b}$ to make $\alpha$ is even or odd.

A loop $\beta$ is said to be a positive or a negative loop in $\bar{a} \bar{b}$ according as the number of negative edges involved in the loop $\beta$ is even or odd. Then $\bar{a} \bar{b}=x^{2 d_{1}+d_{2}}$, where $d_{1}$ is the number of positive loops and $d_{2}$ is the number of negative loops. Then $\bar{D}_{n}(x)$ is a finite dimensional algebra.

For example,

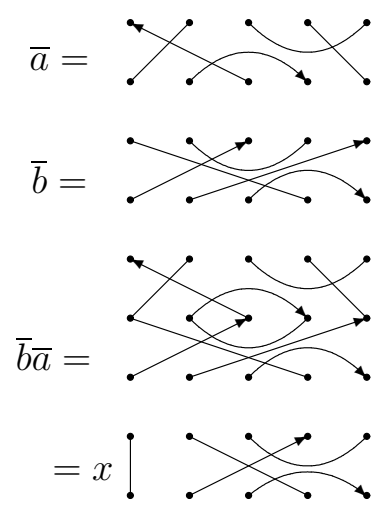

Let $\widetilde{\Gamma}_{n, r}=\bigcup_{f=0}^{\left[\frac{n}{2}\right]} \bar{\Gamma}_{f, r}$, where $\bar{\Gamma}_{f, r}=\left\{\lambda|\lambda \vdash 2(n-2 f)+| \delta_{r} \mid\right.$ whose 2-core is $\delta_{r}, \delta_{r}=$ $(r, r-1, \ldots, 1,0)\}$ for fixed $r \geq 0$. Let $\bar{B}$ be the Bratteli diagram whose vertices on the $k^{\text {th }}$ floor are members of $\widetilde{\Gamma}_{n, r}$. Note that $0^{\text {th }}$ floor contains precisely the core $\delta_{r}$. The $i^{\text {th }}$ vertex on the $k^{\text {th }}$ floor and $j^{\text {th }}$ vertex on the $(k-1)^{\text {th }}$ floor are joined whenever the latter is obtained from the former by removing a rim 2-hook.

Definition 2.21. [PK] An up-down path $p$ in $\bar{B}$ is defined as the sequence of partitions in $\widetilde{\Gamma}_{n, r}$ starting from the $0^{\text {th }}$ floor to the $1^{\text {th }}$ floor. i.e. it can be considered as $p=$ $\left[\delta_{r}=\lambda^{0}, \lambda^{1}, \ldots, \lambda^{n}\right]$ where $\lambda^{i}$ is obtained from $\lambda^{i-1}$ either by adding or removing of only one rim 2-hook. 
Let $\left|\widetilde{\Omega}_{n}\right|$ denote the number of up-down paths ending at the $n^{\text {th }}$ floor. $\left|\widetilde{\Omega}_{n, \lambda}\right|$ denotes the number of up-down paths ending at $\lambda$ in the $n^{\text {th }}$ floor.

The paths belong to $\widetilde{\Omega}_{n, \lambda}$ are called the $d$-vacillating tableau of shape $\lambda, \lambda \in \widetilde{\Gamma}_{n, r}$.

\section{The Robinson-Schensted correspondence for the signed Brauer algebras}

\subsection{The Robinson-Schensted correspondence using bi-domino tableau}

In this section, we define a Robinson-Schensted algorithm for the signed Brauer algebra which gives the correspondence between the signed Brauer diagram $d$ and the pairs of standard bi-dominotableaux of shape $\lambda=\left(\lambda_{1}, \lambda_{2}\right)$ with $\lambda_{1}=\left(2^{2 f}\right), \lambda_{2} \in \bar{\Gamma}_{f, r}$, for fixed $r \geq 0$ and $0 \leq f \leq\left[\frac{n}{2}\right]$.

Definition 3.1. A domino in which all the nodes are filled with same number from the set $A=\{1,2, \cdots n\}$ is defined as a tablet.

Definition 3.2. A bipartition $\nu$ of $2 n$ will be an ordered pair of partitions $\left(\nu^{(1)}, \nu^{(2)}\right)$ where $\nu^{(1)}=\left(2^{2 f}\right)$ and $\nu^{(2)} \in \bar{\Gamma}_{f, r}$, for fixed $r \geq 0$.

Definition 3.3. A standard horizontal block is defined as the block consisting of two horizontal tablets $d^{(1)}, d^{(2)}$ one above the other such that $d^{(1)}<d^{(2)}$. i.e. $\frac{d^{(1)}}{d^{(2)}} d^{(2)}$. We call $d^{(1)}$ as the first tablet of the horizontal block and $d^{(2)}$ as the second tablet of the horizontal block. We call horizontal block as positive block.

Definition 3.4. A standard vertical block is defined as the block consisting of two vertical

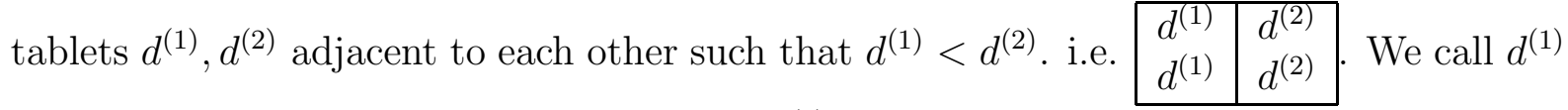
as the first tablet of the vertical block and $d^{(2)}$ as the second tablet of the vertical block. We call vertical block as negative block.

Definition 3.5. A block tableau is a tableau consisting either of the horizontal block or the vertical block.

Definition 3.6. A column standard block tableau is a block tableau if the head nodes of the first tablets of each block are increasing read from top to bottom.

Definition 3.7. A standard tableau is a tableau consisting of tablets such that the head nodes of the tablets are increasing along the rows and increasing along the columns.

Definition 3.8. Let $\nu^{(1)}, \nu^{(2)}$ be as in Definition 3.2. A $\nu$-bi-dominotableau $t$ is standard if $t^{(1)}$ is the column standard block tableau and $t^{(2)}$ is the standard tableau. The collection of standard $\nu$-bi-dominotableaux will be denoted by $\operatorname{Std}(\nu)$. 
Definition 3.9. Given a signed Brauer diagram $d \in \bar{V}_{n}$, we may associate a triple $\left[d_{1}, d_{2}, w\right]$ such that

$$
\begin{aligned}
d_{1}= & \left\{\left(i, d_{1}(i), c\left(d_{1}(i)\right)\right) \mid \text { the edge joining the vertices } i \text { and } d_{1}(i)\right. \text { in the first row with } \\
& \left.\operatorname{sign} c\left(d_{1}(i)\right)\right\} \\
= & \left\{\left(i_{1}, d_{1}\left(i_{1}\right), c\left(d_{1}\left(i_{1}\right)\right)\right),\left(i_{2}, d_{1}\left(i_{2}\right), c\left(d_{1}\left(i_{2}\right)\right)\right), \ldots,\left(i_{f}, d_{1}\left(i_{f}\right), c\left(d_{1}\left(i_{f}\right)\right)\right)\right\} \\
d_{2}= & \left\{\left(j, d_{2}(j), c\left(d_{2}(j)\right)\right) \mid \text { the edge joining the vertices } j \text { and } d_{2}(j)\right. \text { in the second row } \\
& \text { with sign } \left.c\left(d_{2}(j)\right)\right\} \\
= & \left\{\left(i_{1}, d_{2}\left(i_{1}\right), c\left(d_{2}\left(i_{1}\right)\right)\right),\left(i_{2}, d_{2}\left(i_{2}\right), c\left(d_{2}\left(i_{2}\right)\right)\right), \ldots,\left(i_{f}, d_{2}\left(i_{f}\right), c\left(d_{2}\left(i_{f}\right)\right)\right)\right\} \\
w= & \{(k, w(k), c(w(k))) \mid \text { the edge joining the vertex } k \text { in the first row and } w(k) \text { in } \\
& \text { the second row with sign } c(w(k))\} \\
= & \left\{\left(i_{1}, w\left(i_{1}\right), c\left(w\left(i_{1}\right)\right)\right),\left(i_{2}, w\left(i_{2}\right), c\left(w\left(i_{2}\right)\right)\right), \ldots,\left(i_{n-2 f}, w\left(i_{n-2 f}\right), c\left(w\left(i_{n-2 f}\right)\right)\right)\right\}
\end{aligned}
$$

such that $i_{1}<i_{2}<\ldots<i_{n-2 f}$ where $f$ is the number of horizontal edges in a row of $d$ and $n-2 f$ is the number of vertical edges in $d$ and $c(x)=$ the sign of the edge joining between $x$ and its preimage, $c(x) \in\{ \pm 1\}$.

Theorem 3.10. The map $d \stackrel{R-S}{\longleftrightarrow}\left[\left(P_{1}(d), P_{2}(d)\right),\left(Q_{1}(d), Q_{2}(d)\right)\right]$ provides a bijection between the set of signed Brauer diagrams $d$ and the pairs of standard $\lambda$-bi-dominotableaux.

Proof. We first describe the map that, given a diagram $d \in \bar{V}_{n}$, produces a pair of bidominotableaux. "d $\stackrel{R-S}{\longleftrightarrow}\left[\left(P_{1}(d), P_{2}(d)\right),\left(Q_{1}(d), Q_{2}(d)\right)\right]^{\prime \prime}$

We construct a sequence of tableaux

$$
\begin{aligned}
& \emptyset=P_{1}^{0}, P_{1}^{1}, \ldots, P_{1}^{f} \\
& \emptyset=Q_{1}^{0}, Q_{1}^{1}, \ldots, Q_{1}^{f} \\
& \emptyset=P_{2}^{0}, P_{2}^{1}, \ldots, P_{2}^{n-2 f} \\
& \emptyset=Q_{2}^{0}, Q_{2}^{1}, \ldots, Q_{2}^{n-2 f}
\end{aligned}
$$

where $f$ is the number of horizontal edges, the edges joining the vertices $\left(x_{1}, x_{2}\right)$ with the sign $c$ are inserted into $P_{1}(d), P_{2}(d), Q_{1}(d)$ and placed in $Q_{2}(d)$ so that $\operatorname{sh} P_{1}^{k}=\operatorname{sh} Q_{1}^{k}$, for all $k$ and $\operatorname{sh} P_{2}^{j}=\operatorname{sh} Q_{2}^{j}$, for all $j$.

Begin with the tableau $P_{1}^{0}=Q_{1}^{0}=\emptyset$ and $P_{2}^{0}=Q_{2}^{0}=t_{0}$, where $t_{0}$ is the tableau of shape $\delta_{r}$, for fixed $r \geq 0$ with entries 0 's. Then recursively define the standard tableau by the following.

$$
\begin{aligned}
& \text { If }\left(l^{\prime}, m^{\prime}\right) \in d_{2} \text { then } P_{1}^{k}=\text { insertion of }(l, m) \text { in } P_{1}^{k-1} \text {. } \\
& \text { If }(l, m) \in d_{1} \text { then } Q_{1}^{k}=\text { insertion of }(l, m) \text { in } Q_{1}^{k-1} \text {. } \\
& \text { If }\left(l, m^{\prime}\right) \in w \text { then } P_{2}^{k}=\text { insertion of } m \text { in } P_{2}^{k-1} \text { and place } l \text { in } Q_{2}^{k-1} \text { where the } \\
& \text { insertion terminates in } P_{2}^{k-1} \text { when } m \text { is inserted. }
\end{aligned}
$$

The operations of insertion and placement will now be described. 
First we give the insertion on $P_{1}(d)$. Let $\left(i_{k}, d_{2}\left(i_{k}\right), c\left(d_{2}\left(i_{k}\right)\right)\right) \in d_{2}$ and $i_{k}, d_{2}\left(i_{k}\right)$ be the elements not in $P_{1}(d)$. To insert $i_{k}, d_{2}\left(i_{k}\right)$ with sign $c\left(d_{2}\left(i_{k}\right)\right)$ into $P_{1}(d)$, we proceed as follows.

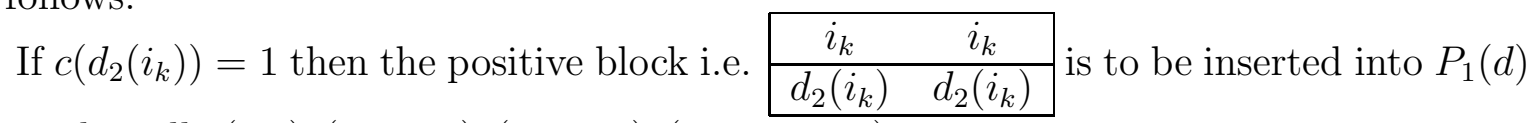
along the cells $(i, j),(i, j+1),(i+1, j),(i+1, j+1)$.

If $c\left(d_{2}\left(i_{k}\right)\right)=-1$ then the negative block i.e. $\beta_{x}=$\begin{tabular}{l|l}
$i_{k}$ & $d_{2}\left(i_{k}\right)$ \\
$i_{k}$ & $d_{2}\left(i_{k}\right)$
\end{tabular} is to be inserted into $P_{1}(d)$ along the cells $(i, j),(i+1, j),(i, j+1),(i+1, j+1)$.

Now place the block containing $i_{k}, d_{2}\left(i_{k}\right)$ below the block containing $i_{k-1}, d_{2}\left(i_{k-1}\right)$. Insertion on $Q_{1}(d)$ is the same as in $P_{1}(d)$.

Insertion on $P_{2}(d)$ is the same as in the case of hyperoctahedral group. We give it here for the sake of completion.

Algorithm BDT

Let $w(x)$ be the element not in $P_{2}(d)$. To insert $w(x)$ in $P_{2}(d)$, we proceed as follows.

If $c(w(x))=1$ then the horizontal tablet $\alpha_{w(x)}=$\begin{tabular}{|l|l|}
$w(x)$ & $w(x)$
\end{tabular} is to be inserted into $P_{2}(d)$ along the cells $(i, j)$ and $(i, j+1)$. The $(i, j+1)$ th cell of $\alpha_{x}$ is called the head node of $\alpha_{w(x)}$ and the $(i, j)$ th cell of $\alpha_{w(x)}$ is called the tail node of $\alpha_{w(x)}$.

If $c(w(x))=-1$ then the vertical tablet $\beta_{w(x)}=\frac{w(x)}{w(x)}$ is to be inserted into $P_{2}(d)$ along the cells $(i, j)$ and $(i+1, j)$. The $(i, j)$ th cell of $\beta_{w(x)}$ is called the head node of $\beta_{w(x)}$ and the $(i+1, j)$ th cell of $\beta_{w(x)}$ is called the tail node of $\beta_{w(x)}$.

If $c(w(x))=1$ then,

A Set row $i:=1$, head node of $\alpha_{w(x)}:=w(x)$ and tail node of $\alpha_{w(x)}:=w(x)$.

B If head node of $\alpha_{w(x)}$ is less than some element of row $i$ then

Let $y_{1}$ be the smallest element of row $i$ greater than $w(x)$ such that the north west most corner of the domino containing $y_{1}$ is in the cell $(i, j)$ and $y_{2}$ be

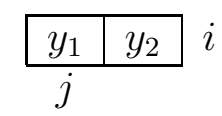
the element in the cell $(i, j+1)$.

2 cases arise,

(BI) tablet containing $y_{1}$ is horizontal i.e., $\alpha_{y_{1}}$.

$$
\left(y_{1}=y_{2}\right)
$$

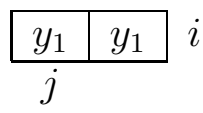

(BII) tablet containing $y_{1}$ is vertical i.e., $\beta_{y_{1}}$.

$$
\left(y_{1} \neq y_{2}\right)
$$

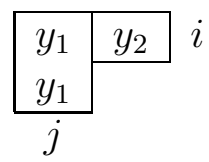

BI If the tablet containing $y_{1}$ is $\alpha_{y_{1}}$ (head node of $\alpha_{y_{1}}$ is in the cell $(i, j+1)$ and the tail node of $\alpha_{y_{1}}$ is in the cell $\left.(i, j)\right)$ i.e. $y_{1}=y_{2}$ then, replace tablet $\alpha_{y_{1}}$ by tablet $\alpha_{w(x)}$. Set tablet $\alpha_{w(x)}:=$ tablet $\alpha_{y_{1}}$, Row $i:=i+1$ and go to B. 
BII If the tablet containing $y_{1}$ is $\beta_{y_{1}}$. (i.e. head node of $\beta_{y_{1}}$ is in the cell $(i, j)$ and the tail node of $\beta_{y_{1}}$ is in the cell $\left.(i+1, j)\right)$ i.e. $y_{1} \neq y_{2}$ then

Let $w_{1}$ be the element in the cell $(i+1, j+1)$.

\begin{tabular}{|c|c|}
\hline$y_{1}$ & $y_{2}$ \\
\cline { 2 - 2 }$y_{1}$ & $w_{1}$ \\
\hline$j$
\end{tabular}

2 cases arise

\begin{tabular}{|c|c|c|}
\hline BIIa $w_{1}=y_{2}$ & $\begin{array}{l}y_{1} \\
y_{1}\end{array}$ & $\begin{array}{l}y_{2} \\
y_{2}\end{array}$ \\
\hline & $j$ & \\
\hline BIIb $w$ & $\begin{array}{l}y_{1} \\
y_{1}\end{array}$ & $\frac{y_{2}}{w_{1}}$ \\
\hline
\end{tabular}

BIIa If $w_{1}=y_{2}$ then replace $w_{1}$ by $y_{1}$ and set tablet $\beta_{w(x)}=y_{2}$ and column $j:=j+2$ and go to $\mathbf{B}^{\prime} .\left(\mathbf{B}^{\prime}\right.$ is the case as in $\mathbf{B}$ by replacing row by column, column by row, positive tablet by negative tablet and negative tablet by positive tablet.)

BIIb If $w_{1} \neq y_{2}$ then let $w_{2}$ be the element in the cell $(i+1, j+2)$. Replace $w_{1}$ and $w_{2}$ by $y_{1}$ and $y_{2}$ respectively, and set $y_{1}:=w_{1}, y_{2}:=w_{2}$, row $i:=i+1$. If $x_{1}=x_{2}$ then set row $i:=i+1$ and go to $\mathbf{B}$ else go to BII.

C Now head node of $\alpha_{w(x)}$ is greater than every element of row $i$ so place the tablet $\alpha_{w(x)}$ at the end of the row $i$ and stop.

If $c(w(x))=-1$ then, replace row by column, column by row, positive tablet by negative tablet and negative tablet by positive tablet in the positive case.

The placement of the tablet of an element in a tableau is even easier than the insertion. Suppose that $Q_{2}(d)$ is a partial tableau of shape $\mu$ and if $k$ is greater than every element of $Q_{2}(d)$, then place the tablet of $k$ in $Q_{2}(d)$ along the cells where the insertion in $P_{2}(d)$ terminates.

To prove " $\left[\left(P_{1}(d), P_{2}(d)\right),\left(Q_{1}(d), Q_{2}(d)\right)\right] \stackrel{R-S}{\longrightarrow} d^{\prime \prime}$. We merely reverse the preceding algorithm step by step. We begin by defining

$$
\left[\left(P_{1}^{f}, P_{2}^{n-2 f}\right),\left(Q_{1}^{f}, Q_{2}^{n-2 f}\right)\right]=\left[\left(P_{1}(d), P_{2}(d)\right),\left(Q_{1}(d), Q_{2}(d)\right)\right]
$$

where $f$ is the number of horizontal edges in a row of $d$ and $n-2 f$ is the number of vertical edges in $d$.

Reverse Algorithm BDT. Assuming that $P_{2}^{k}$ and $Q_{2}^{k}$ has been constructed we will find the pair $\left(x_{k}, w\left(x_{k}\right), c\left(w\left(x_{k}\right)\right)\right)$ and $\left[\left(P_{1}^{k-1}, P_{2}^{k-1}\right),\left(Q_{1}^{k-1}, Q_{2}^{k-1}\right)\right]$.

Find the cells containing the tablet of $x_{k}$ in $Q_{2}^{x_{k}}$.

2 cases arise,

$\dagger$ The cells containing tablet of $x_{k}$ in $Q_{2}^{x_{k}}$ are $(i, j-1)$ and $(i, j)$

$\ddagger$ The cells containing tablet of $x_{k}$ in $Q_{2}^{x_{k}}$ are $(i-1, j)$ and $(i, j)$ 
case $\dagger$ If the cells containing tablet of $x_{k}$ in $Q_{2}^{x_{k}}$ are $(i, j-1)$ and $(i, j)$, then since this is the largest element whose tablet appears in $Q_{2}^{x_{k}}, P_{2}^{i, j-1}, P_{2}^{i, j}$ must have been the last element to be placed in the construction of $P_{2}^{x_{k}}$.

We can now use the following procedure to delete $P_{2}^{i, j-1}, P_{2}^{i, j}$ from $P_{2}(d)$. For convenience, we assume the existence of an empty zeroth row above the first row of $P_{2}^{x_{k}}$ and empty zeroth column to the left of the first column of $P_{2}^{x_{k}}$.

Set $x_{1}:=P_{2}^{i, j-1}, x_{2}:=P_{2}^{i, j}$ and erase $P_{2}^{i, j-1}, P_{2}^{i, j}$.

2 cases arise,

(A) $x_{1}=x_{2}$

(B) $x_{1} \neq x_{2}$

case $\mathbf{A}$ If $x_{1}=x_{2}$ then

case AI Set head node of $\alpha_{x}:=x_{2}$ and tail node of $\alpha_{x}:=x_{1}$ and Row $i:=(i-1)$ th row of $P_{2}^{x_{k}}$.

case AII If Row $i$ is not the zeroth row of $P_{2}^{x_{k}}$ then

Let $y$ be the largest element of Row $i$ smaller than $w(x)$ which is in the cell $(i, l)$

(2 cases arise,

(AIIa) the tablet containing $y$ is $\alpha_{y}$

(AIIb) the tablet containing $y$ is $\beta_{y}$ )

case AIIa If the tablet containing $y$ is $\alpha_{y}$ then replace tablet $\alpha_{y}$ by tablet $\alpha_{x}$. Set tablet $\alpha_{x}:=$ tablet $\alpha_{y}$, Row $i:=i-1$ and goto AII

case AIIb If the tablet containing $y$ is $\beta_{y}$ then

Let $z$ be the element in the cell $(i, l-1)$ and replace tail node of $\beta_{y}$ and $z$ by tablet $\alpha_{x}$. Set $x_{1}:=z$ and $x_{2}:=$ tail node of $\beta_{y}$ and go to $\mathbf{B}$.

case AIII Now the tablet $\alpha_{x}$ has been removed from the first row, so set $w\left(x_{k}\right):=x$ and $c\left(w\left(x_{k}\right)\right)=1$.

case $\mathbf{B}$ If $x_{1} \neq x_{2}$ then

2 cases arise,

(B1) the tablet containing $x_{1}$ is $\beta_{x_{1}}$

(B2) the tablet containing $x_{1}$ is $\alpha_{x_{1}}$

case B1 If the tablet containing $x_{1}$ is $\beta_{x_{1}}$ then

replace head node of $\beta_{x_{1}}$ by tail node of $\beta_{x_{2}}$. Set tablet $\beta_{w(x)}:=$ tablet $\beta_{x_{1}}$ and Column $j:=j-2$ and go to $\mathbf{A}^{\prime} \mathbf{I I}$. ( $\mathbf{A}^{\prime} \mathbf{I I}$ is the case as in AII by replacing row by column, column by row, positive tablet by negative tablet and negative tablet by positive tablet.)

case B2 If the tablet containing $x_{1}$ is $\alpha_{x_{1}}$ then

replace the elements in the cell $(i-1, j-2)$ and $(i-1, j-1)$ by head node of $\alpha_{x_{1}}$ and tail node of $\beta_{x_{2}}$ respectively. Set $x_{1}:=$ the element in the cell 
$(i-1, j-2), x_{2}:=$ the element in the cell $(i-1, j-1)$ and if $x_{1}=x_{2}$ then Row $i:=i-1$ and go to $\mathbf{A}$ else Column $j=j-1$ and go to $\mathbf{B}$.

case $\ddagger$ follows as in case $\dagger$ by replacing row by column, column by row, positive tablet by negative tablet and negative tablet by positive tablet.

It is easy to see that $P_{2}^{x_{k}-1}$ is $P_{2}^{x_{k}}$ after the deletion process complete and $Q_{2}^{x_{k}-1}$ is $Q_{2}^{x_{k}}$ with the tablet of $x_{k}$ erased. Continuing in this way, we recover all the elements of $w$ in reverse order.

We are yet to find the elements in $d_{1}, d_{2}$.

We may recover the elements of $d_{2}$ such that the pair $\left(x_{k}, d_{2}\left(x_{k}\right), c\left(d_{2}\left(x_{k}\right)\right)\right)$ is the block in the cells $((2 k-1,1),(2 k-1,2),(2 k, 1),(2 k, 2))$ of $P_{1}(d)$, for every $k$ and the $c\left(d_{2}\left(x_{k}\right)\right)=1\left(c\left(d_{2}\left(x_{k}\right)\right)=-1\right)$ if the block is positive block (negative block).

Similarly, we may recover the elements of $d_{1}$ such that the pair $\left(x_{k}, d_{1}\left(x_{k}\right), c\left(d_{1}\left(x_{k}\right)\right)\right)$ is the element in the cells $((2 k-1,1),(2 k-1,2),(2 k, 1),(2 k, 2))$ of $Q_{1}(d)$, for every $k$ and the $c\left(d_{1}\left(x_{k}\right)\right)=1\left(c\left(d_{1}\left(x_{k}\right)\right)=-1\right)$ if the block is positive block (negative block).

Thus we recover the triple $d_{1}, d_{2}, w$ from the pair of bi-dominotableaux

$$
\left[\left(P_{1}(d), P_{2}(d)\right),\left(Q_{1}(d), Q_{2}(d)\right)\right]
$$

Hence $\left[\left(P_{1}(d), P_{2}(d)\right),\left(Q_{1}(d), Q_{2}(d)\right)\right] \stackrel{R-S}{\longrightarrow} d$ which completes the proof.

Example 3.11. Let

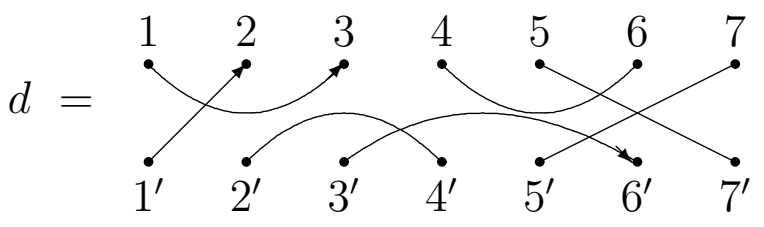

$d_{1}=\{(1,3,-1),(4,6,1)\} d_{2}=\left\{\left(2^{\prime}, 4^{\prime}, 1\right),\left(3^{\prime}, 6^{\prime},-1\right)\right\} w=\left\{\left(2,1^{\prime},-1\right),\left(5,7^{\prime}, 1\right),\left(7,5^{\prime}, 1\right)\right\}$

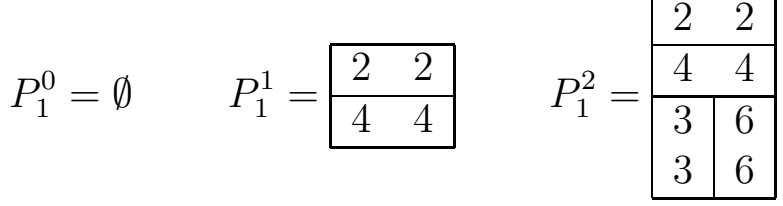

$$
\begin{aligned}
& Q_{1}^{0}=\emptyset \quad Q_{1}^{1}=\begin{array}{lll}
1 & 3 \\
1 & 3
\end{array} \quad Q_{1}^{2}=\begin{array}{ll}
1 & 3 \\
\hline 4 & 4 \\
\hline 6 & 6 \\
\hline
\end{array}
\end{aligned}
$$

For the core $\delta_{r}, r=0$, we have

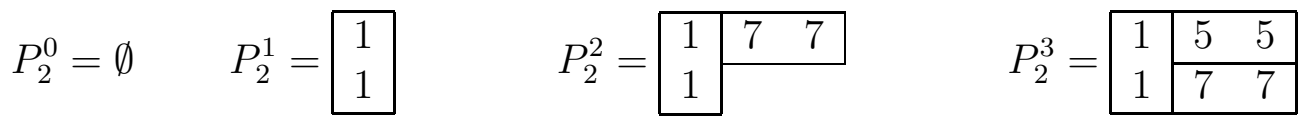




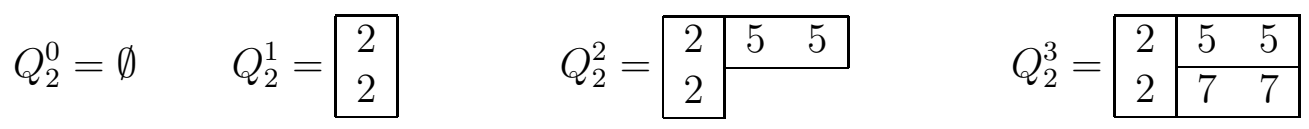

Thus

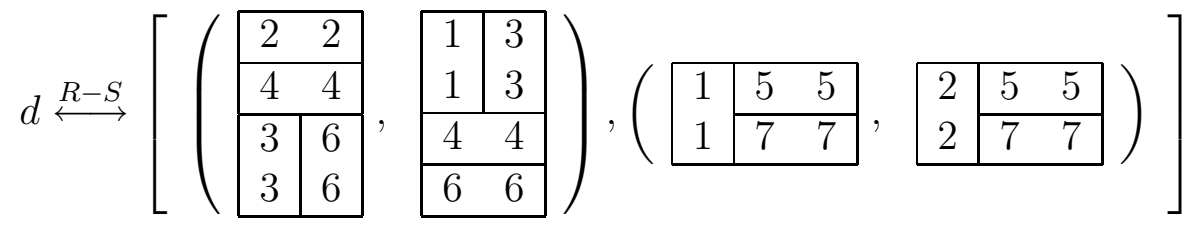

For the core $\delta_{r}, r=(2,1)$, the above diagram gives

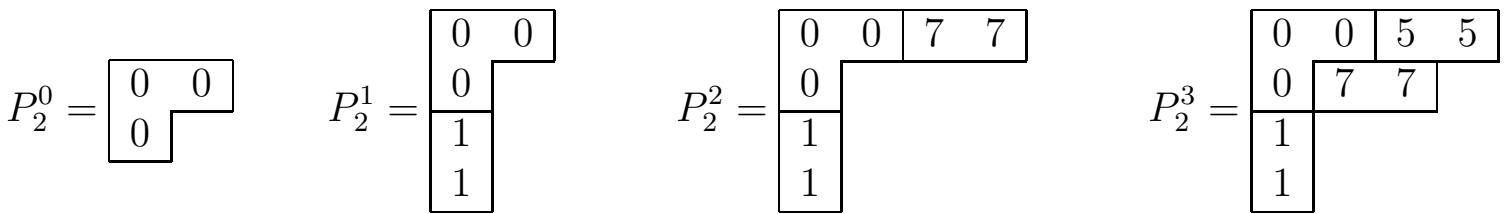

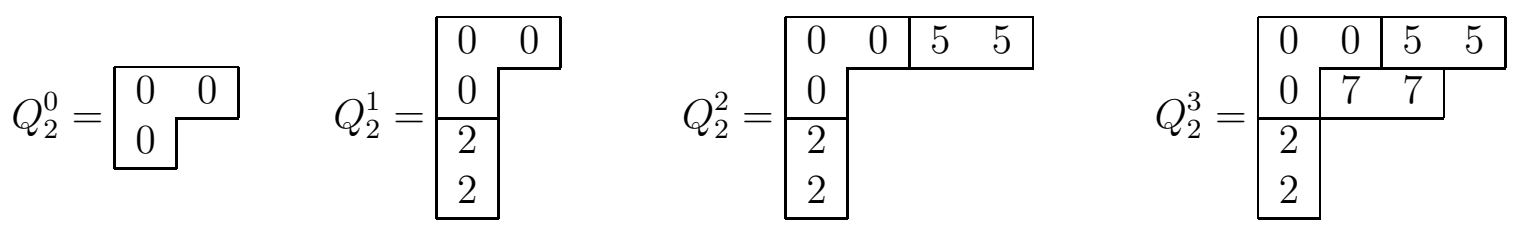

Thus

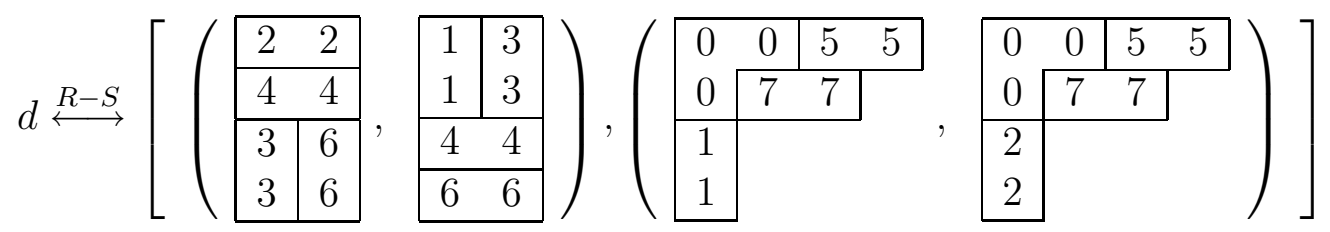

Remark 3.12. If $d \in \bar{V}_{n}$ has no horizontal edges $\left(d_{1}=d_{2}=\emptyset\right)$ then $d$ is an element in the hyperoctahedral group. Hence

$$
P_{1}(d)=\emptyset, \quad P_{2}(d)=P, \quad Q_{1}(d)=\emptyset, \quad Q_{2}(d)=Q
$$

where $P_{1}(d), Q_{1}(d)$ are the column standard block tableau and $P_{2}(d), Q_{2}(d)$ are the standard tableau constructed by the above insertion and $P, Q$ are the tableaux of shape $\lambda \in \bar{\Gamma}_{0, r}$, for fixed $r \geq 0$ constructed by the Robinson-Schensted correspondence for the hyperoctahedral group of type $B_{n}$.

Corollary 3.13. The map $d \stackrel{R-S}{\longleftrightarrow}\left[\left(P_{1}(d), P_{2}(d)\right),\left(Q_{1}(d), Q_{2}(d)\right)\right]$ provides a bijection between the set of Brauer diagrams $d$ and the pairs of standard bitableaux of shape $\lambda=\left(\lambda_{1}, \lambda_{2}\right)$ with $\lambda_{1}=\left(2^{f}\right), \lambda_{2} \in \Gamma_{f}$ where $\Gamma_{f}=\{\lambda \mid \lambda \vdash n-2 f\}$ and $0 \leq f \leq\left[\frac{n}{2}\right]$. 
Proof. Each signed Brauer diagram is a Brauer diagram if and only if each edge is labelled by a positive sign and the proof follows by replacing each positive tablet $x$ by the node $x$ in Theorem 3.10.

Definition 3.14. The flip of any signed Brauer diagram $d$ is the diagram of $d$ reflected over its horizontal axis, which is denoted by flip $(d)$.

Proposition 3.15. Let $d \in \bar{V}_{n}$. If $d \longrightarrow\left[\left(P_{1}(d), P_{2}(d)\right),\left(Q_{1}(d), Q_{2}(d)\right)\right]$. Then

$$
\text { flip }(d) \longrightarrow\left[\left(Q_{1}(d), Q_{2}(d)\right),\left(P_{1}(d), P_{2}(d)\right)\right]
$$

where $P_{1}(d), Q_{1}(d)$ are the column standard block tableaux and $P_{2}(d), Q_{2}(d)$ are the standard tableaux constructed by the above insertion.

Proof. Suppose $d \in \bar{V}_{n}$, then we can recover the triple $\left[d_{1}, d_{2}, w\right]$ by the Definition 3.9. By the definition flip $(d)$ has the triple $\left[d_{2}, d_{1}, w^{-1}\right]$. Hence the proof follows by Proposition 2.13 .

\subsection{The Robinson-Schensted correspondence using vacillating tableau}

In this section, we follow the Robinson-Schensted correspondence using vacillating tableau for the Partition algebras in [HL], to construct the Robinson-Schensted correspondence for the signed Brauer algebras.

Let us denote by $\widetilde{T}_{n}(\lambda)$ the set of $d$-vacillating tableaux of shape $\lambda$ and length $n+1$. Thus $\left|\widetilde{\Omega}_{n, \lambda}\right|=\left|\widetilde{T}_{n}(\lambda)\right|$.

To give a combinatorial proof of

$$
2^{n}(2 n) ! !=\sum_{\lambda \in \widetilde{\Gamma}_{f, r}}\left|\widetilde{\Omega}_{n, \lambda}\right|^{2} \quad \text { for fixed } r \geq 0 \text { and } 0 \leq f \leq\left[\frac{n}{2}\right]
$$

we find a bijection of the form

$$
\bar{V}_{n} \longleftrightarrow \bigsqcup_{\lambda \in \bar{\Gamma}_{f, r}, r \geq 0} \widetilde{T}_{n}(\lambda) \times \widetilde{T}_{n}(\lambda) \quad \text { for fixed } r \geq 0 \text { and } 0 \leq f \leq\left[\frac{n}{2}\right]
$$

by constructing a function that takes a signed Brauer diagram $d \in \bar{V}_{n}$ and produces a pair $\left(P_{\lambda}, Q_{\lambda}\right)$ of $d$-vacillating tableaux.

We will draw diagrams $d \in \bar{V}_{n}$ using a standard representation as a single row representation with $2 n$ vertices labeled $1,2, \ldots, 2 n$, where we relabel vertex $j^{\prime}$ with the label $(2 n-j+1)$. We draw the edges of the standard representation of $d \in \bar{V}_{n}$ in a specific way such that: Connect the vertices $i$ and $j$ for $i \leq j$ if and only if $i$ and $j$ are related in $d$. In this way, connect each vertex. We label each positive edge of the diagram $d$ with $(2 n-m+1)$ where $m$ is the right vertex and each negative edge of the diagram $d$ with $-(2 n-m+1)$. 
Definition 3.16. The insertion sequence of a diagram $d \in \bar{V}_{n}$ to be the sequence $I^{(d, n)}=$ $\left(I_{j}^{(d, n)}\right)$ indexed by $j$ in the sequence $1,2, \ldots, 2 n$, where

$I_{j}^{(d, n)}=\left\{\begin{aligned} a, & \text { if vertex } j \text { is left endpoint of positive edge } a \\ -a, & \text { if vertex } j \text { is left endpoint of negative edge } a \\ (a, \emptyset), & \text { if vertex } j \text { is right endpoint of either a positive or a negative edge } a\end{aligned}\right.$

Thus the insertion sequence is \begin{tabular}{|c|c|c|c|c|}
\hline$j$ & 1 & 2 & $\cdots$ & $2 n$ \\
\cline { 2 - 6 }$\left(I_{j}^{(d, n)}\right.$ & $x_{1}$ & $x_{2}$ & $\cdots$ & $x_{2 n}$ \\
\hline
\end{tabular}

Proposition 3.17. $d \in \bar{V}_{n}$ is completely determined by its insertion sequence.

Proof. The proof is the same as in [HL], we give it here for the sake of completion. Since the insertion sequence of a diagram $d$ completely determines the edges, the proof follows.

Note. $I_{j}^{(d, n)} \stackrel{d}{\longleftarrow} T^{(j-1)}$ means the domino containing $I_{j}^{(d, n)}$ is deleted from $T^{(j-1)}$ and $I_{j}^{(d, n)} \stackrel{R-S}{\longrightarrow} T^{(j-1)}$ means the domino containing $I_{j}^{(d, n)}$ is to be inserted in $T^{(j-1)}$ using algorithm BDT in theorem 3.10.

For $d \in \bar{V}_{n}$ with insertion sequence $I^{(d, n)}=\left(I_{j}^{(d, n)}\right)$, we will produce a pair $\left(P_{\lambda}, Q_{\lambda}\right)$ of $d$-vacillating tableaux.

Let $T^{(0)}$ be the tableau of shape $\delta_{r}$ with entries 0 's. Then recursively define standard tableau $T^{(j+1)}$ by

$$
T^{(j+1)}= \begin{cases}I_{j+1}^{(d, n)} \stackrel{R-S}{\longrightarrow} T^{(j)} & \text { if } I_{j+1}^{(d, n)}=x_{k} \\ I_{j+1}^{(d, n)} \stackrel{\mathrm{d}}{\longleftarrow} T^{(j)} & \text { if } I_{j+1}^{(d, n)}=\left(x_{k}, \emptyset\right) .\end{cases}
$$

Let $\lambda^{(i)}$ be the shape of $T^{(i)}$. Define

$$
\begin{aligned}
Q_{\lambda} & =\left(\delta_{r}, \lambda^{(1)}, \ldots, \lambda^{(n)}\right) \in \widetilde{T}_{n}(\lambda) \\
P_{\lambda} & =\left(\lambda^{(2 n)}, \ldots, \lambda^{(n)}\right) \in \widetilde{T}_{n}(\lambda)
\end{aligned}
$$

In this way, we associate a pair of $d$-vacillating tableaux $\left(P_{\lambda}, Q_{\lambda}\right)$ to $d \in \bar{V}_{n}$ which we denote by $d \underset{\bar{V}_{n}}{\stackrel{R-S}{\longrightarrow}}\left(P_{\lambda}, Q_{\lambda}\right)$.

Theorem 3.18. The map $d \underset{\bar{V}_{n}}{\stackrel{R-S}{\longrightarrow}}\left(P_{\lambda}, Q_{\lambda}\right)$ provides a bijection between the set of signed Brauer diagrams $\bar{V}_{n}$ and the pairs of d-vacillating tableaux of shape $\lambda \in \bar{\Gamma}_{f, r}$, for fixed $r \geq 0$ and $0 \leq f \leq\left[\frac{n}{2}\right]$. i.e.

$$
\bar{V}_{n} \longleftrightarrow \bigsqcup_{\lambda \in \bar{\Gamma}_{f, r}, r \geq 0} \widetilde{T}_{n}(\lambda) \times \widetilde{T}_{n}(\lambda)
$$


Proof. The proof is same as in [HL], we give it here for the sake of completion. We prove the theorem by constructing the inverse of $d \underset{\bar{V}_{n}}{\stackrel{R-S}{\longrightarrow}}\left(P_{\lambda}, Q_{\lambda}\right)$. First we use $Q_{\lambda}$ followed by $P_{\lambda}$ in reverse order to construct the sequence $\lambda^{(1)}, \lambda^{(2)}, \ldots, \lambda^{(2 n)}$. We initialize $T^{(2 n)}=\emptyset$.

We now show how to construct $T^{(i)}$ and $I_{i+1}^{(d, n)}$ so that $T^{(i+1)}=\left(I_{i+1}^{(d, n)} \stackrel{R-S}{\longrightarrow} T^{(i)}\right)$. If $\lambda^{(i+1)} / \lambda^{(i)}$ is a tablet $a$, and we use reverse algorithm BDT on the value in the tablet $a$ to produce $T^{(i)}$ and $I_{i+1}^{(d, n)}$ such that $T^{(i+1)}=\left(I_{i+1}^{(d, n)} \stackrel{R-S}{\longrightarrow} T^{(i)}\right)$. Since we remove the value in position $a$ by using reverse Robinson-Schensted algorithm, we know that $T^{(i)}$ has shape $\lambda^{(i)}$.

We then show how to construct $T^{(i)}$ and $I_{i+1}^{(d, n)}$ so that $T^{(i+1)}=\left(I_{i+1}^{(d, n) \stackrel{d}{\longleftarrow}} T^{(i)}\right)$. If $\lambda^{(i)} / \lambda^{(i+1)}$ is a tablet $a$. Let $T^{(i)}$ be the tableau of shape $\lambda^{(i)}$ with the same entries as $T^{(i+1)}$ and having the entry $2 n-i$ in tablet $a$. Let $I_{i+1}^{(d, n)}=2 n-i$. At any given step $i, 2 n-i$ is the largest domino added to the tableau thus far, so $T^{(i)}$ is standard. Furthermore, $T^{(i+1)}=\left(I_{i+1}^{(d, n)} \stackrel{d}{\longleftarrow} T^{(i)}\right)$, since $I_{i+1}^{(d, n)}=2 n-i$ is already in the rim hook and thus simply delete it.

This iterative process will produce $I_{2 n}^{(d, n)}, I_{2 n-1}^{(d, n)}, \ldots, I_{1}^{(d, n)}$ which completely determines $d$. By the way we have constructed $d$, we have $d \underset{\bar{V}_{n}}{\stackrel{R-S}{\longrightarrow}}\left(P_{\lambda}, Q_{\lambda}\right)$.

Example 3.19. Let $d$ be the diagram as in Example 3.11. Then the single row representation of $d$ is

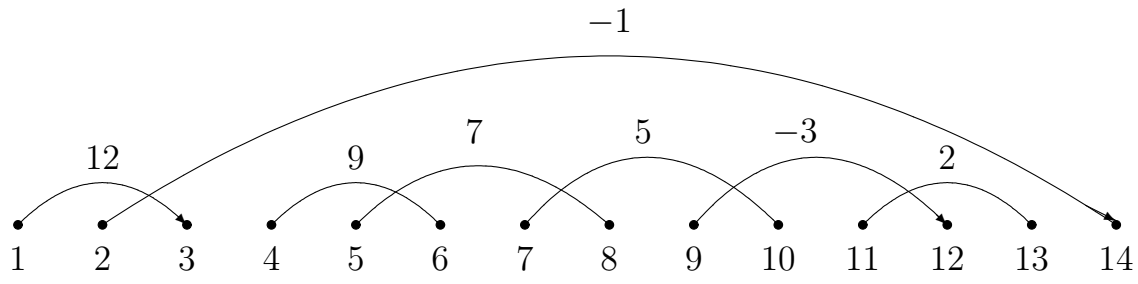

and the insertion sequence is

\begin{tabular}{|c|c|c|c|c|c|c|c|c|}
\hline$j$ & 1 & 2 & 3 & 4 & 5 & 6 & 7 & 8 \\
\hline$I_{j}^{(d, n)}$ & 12 & -1 & $(12, \emptyset)$ & 9 & 7 & $(9, \emptyset)$ & 5 & $(7, \emptyset)$ \\
\hline
\end{tabular}

\begin{tabular}{|c|c|c|c|c|c|c|}
\hline$j$ & 9 & 10 & 11 & 12 & 13 & 14 \\
\hline$I_{j}^{(d, n)}$ & -3 & $(5, \emptyset)$ & 2 & $(-3, \emptyset)$ & $(2, \emptyset)$ & $(-1, \emptyset)$ \\
\hline
\end{tabular}

For the core $\delta_{r}, r=0$, we have

$\begin{array}{lllllll}j & I_{j}^{(d, n)} & T^{(j)} & \frac{j}{(d, n)} & & T^{(j)} \\ 0 & \emptyset & 14 \quad(-1, \emptyset) \stackrel{\mathrm{d}}{\longleftarrow} \emptyset\end{array}$




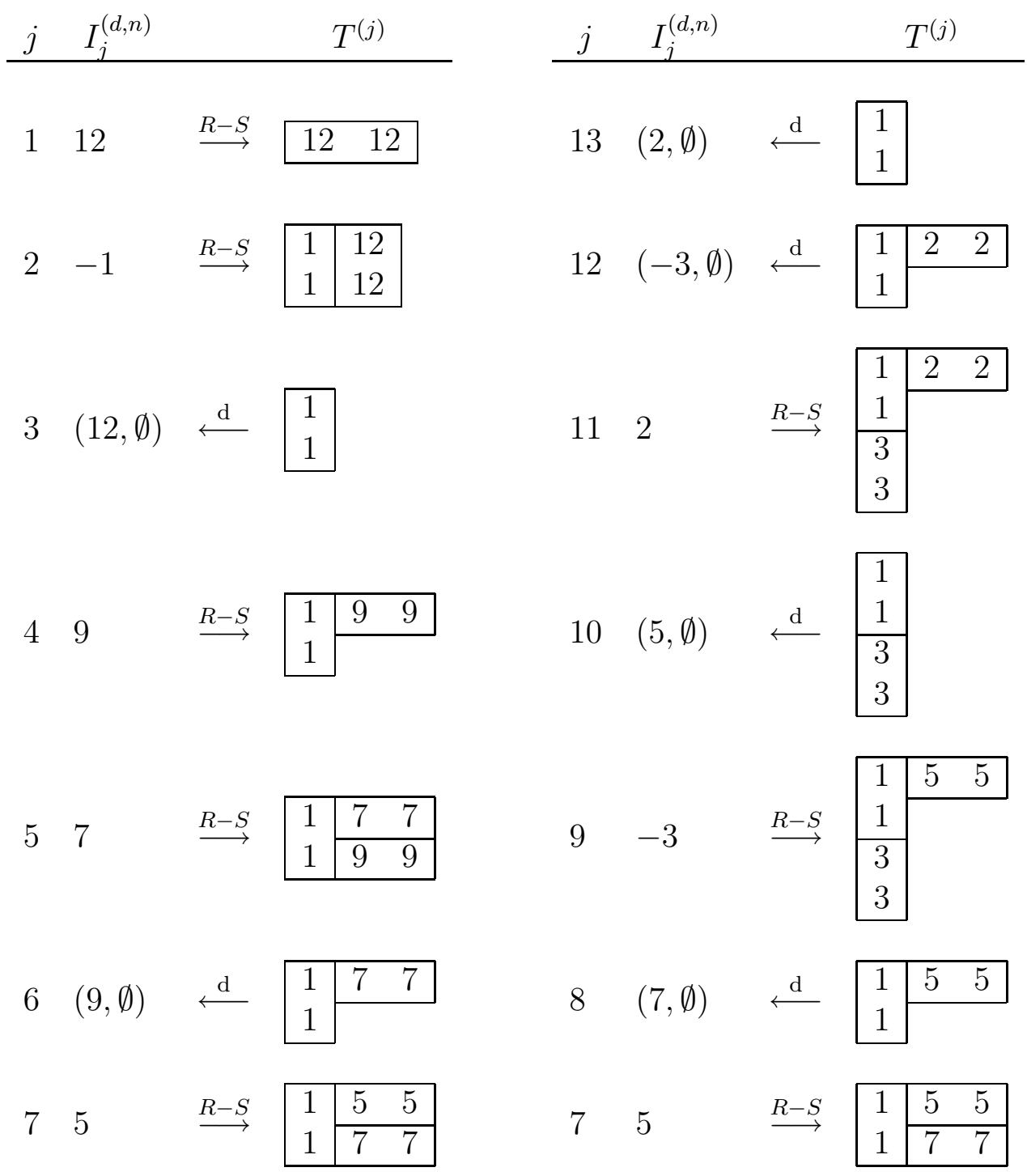

Hence $d \underset{\bar{V}_{n}}{\stackrel{R-S}{\longrightarrow}}\left(P_{\lambda}, Q_{\lambda}\right)$ where

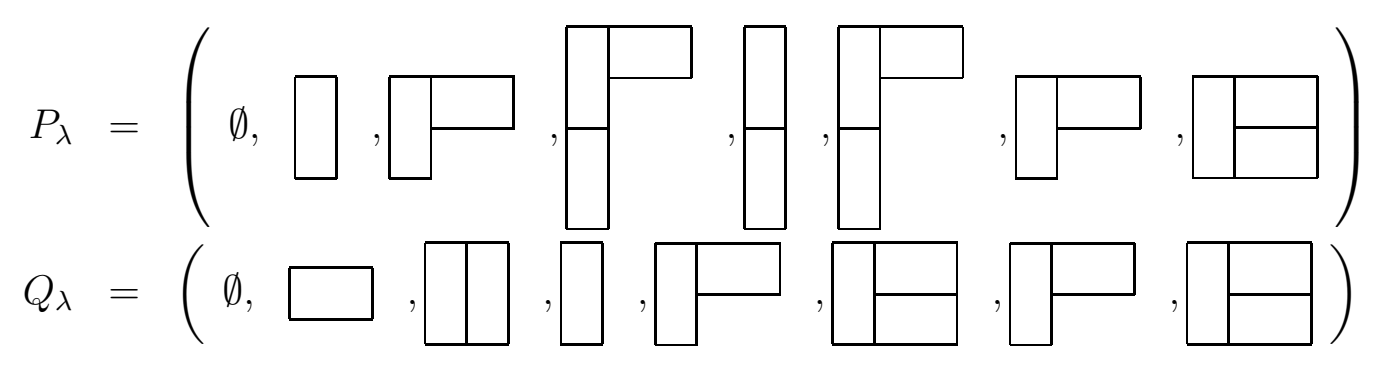

Remark 3.20.

1. Replace the domino as a node in the above procedure, we get the Robinson-Schensted correspondence for the Brauer algebra, which gives the same vacillating tableau as in [DS, HL, Ro1, Ro2, Su]. 
2. We can pass from vacillating tableau to the bi-domino tableau by the following procedure:

Let $\left(P_{\lambda}, Q_{\lambda}\right)$ be the vacillating tableau obtained using the Robinson-Schensted correspondence for the vacillating tableau.

If a positive (negative) domino is added at the $i^{\text {th }}$ step in $P_{\lambda}$ then put $i$ in that domino. If a domino is removed at the $i^{\text {th }}$ step in a vacillating tableau $P_{\lambda}$ then perform reverse algorithm BDT in Theorem 3.10. A number $j$ with positive or negative sign is uninserted.

Now add the positive or negative domino block in $P_{1}(d)$. The final tableau obtained using the above procedure is $P_{1}(d)$. Similarly, construct $Q_{1}(d)$ and $Q_{2}(d)$ using $Q_{\lambda}$.

\section{Applications of Robinson-Schensted correspondence for the signed Brauer algebra using bidomino tableau}

\subsection{The Knuth relations}

In this section, we derive the Knuth relations for the signed Brauer algebra by using the Robinson-Schensted correspondence for the standard bi-dominotableau whose 2-core is $\delta_{r}, r \geq n-1$.

Definition 4.1. A generalized signed permutation is a two-line array of integers

$$
x=\left(\begin{array}{cccc}
i_{1} & i_{2} & \cdots & i_{n} \\
\varepsilon_{x_{1}} x_{1} & \varepsilon_{x_{2}} x_{2} & \cdots & \varepsilon_{x_{n}} x_{n}
\end{array}\right)
$$

where $\varepsilon_{x_{i}} \in\{ \pm 1\}, \forall i$ whose column are in lexicographic order, with the top entry taking precedence and $x_{l} \neq x_{m}, \forall l, m$. The set of all generalized signed permutations is denoted by $\mathcal{G S P}(n)$

Proposition 4.2. If $x \in \mathcal{G S P}(n)$ then $P\left(x^{-1}\right)=Q(x)$ and $Q\left(x^{-1}\right)=P(x)$ where $P(x)$, $P\left(x^{-1}\right), Q(x), Q\left(x^{-1}\right)$ be the standard tableaux of shape $\lambda \in \bar{\Gamma}_{0, r}$, for fixed $r \geq n-1$

Proof. The proof is as in Proposition 2.13.

Definition 4.3. The generalized signed permutations $x$ and $y$ differ by a Knuth relation of first kind, denoted by $x \stackrel{\widetilde{1}}{\sim} y$ if

$$
\begin{aligned}
& x=\varepsilon_{x_{1}} x_{1} \ldots \varepsilon_{x_{i-1}} x_{i-1} \varepsilon_{x_{i}} x_{i} \varepsilon_{x_{i+1}} x_{i+1} \ldots \varepsilon_{x_{n}} x_{n} \text { and } \\
& y=\varepsilon_{x_{1}} x_{1} \ldots \varepsilon_{x_{i-1}} x_{i-1} \varepsilon_{x_{i+1}} x_{i+1} \varepsilon_{x_{i}} x_{i} \ldots \varepsilon_{x_{n}} x_{n}
\end{aligned}
$$

such that $x_{i}<x_{i-1}<x_{i+1}$ and $\varepsilon_{x_{i-1}}=\varepsilon_{x_{i}}=\varepsilon_{x_{i+1}}$.

They differ by a Knuth relation of second kind, denoted by $x \underset{\sim}{\sim} y$ if 


$$
\begin{aligned}
x & =\varepsilon_{x_{1}} x_{1} \ldots \varepsilon_{x_{i}} x_{i} \varepsilon_{x_{i+1}} x_{i+1} \varepsilon_{x_{i-1}} x_{i-1} \ldots \varepsilon_{x_{n}} x_{n} \text { and } \\
y & =\varepsilon_{x_{1}} x_{1} \ldots \varepsilon_{x_{i+1}} x_{i+1} \varepsilon_{x_{i}} x_{i} \varepsilon_{x_{i-1}} x_{i-1} \ldots \varepsilon_{x_{n}} x_{n}
\end{aligned}
$$

such that $x_{i}<x_{i-1}<x_{i+1}$ and $\varepsilon_{x_{i-1}}=\varepsilon_{x_{i}}=\varepsilon_{x_{i+1}}$.

They differ by a Knuth relation of third kind, denoted by $x \stackrel{\widetilde{3}}{\sim} y$ if

$$
x=\varepsilon_{x_{1}} x_{1} \ldots \varepsilon_{x_{i}} x_{i} \varepsilon_{x_{i+1}} x_{i+1} \ldots \varepsilon_{x_{n}} x_{n} \text { and } y=\varepsilon_{x_{1}} x_{1} \ldots \varepsilon_{x_{i+1}} x_{i+1} \varepsilon_{x_{i}} x_{i} \ldots \varepsilon_{x_{n}} x_{n}
$$

such that $\varepsilon_{x_{i}}=-\varepsilon_{x_{i+1}}$.

The two permutations are Knuth equivalent, denoted by $x \stackrel{\widetilde{K}}{\sim} y$ if there is a sequence of permutations such that

$$
x=z_{1} \stackrel{i}{\sim} z_{2} \stackrel{j}{\sim} \ldots \stackrel{l}{\sim} z_{k}=y \text { where } i, j, \ldots, l \in\{\widetilde{1}, \widetilde{2}, \widetilde{3}\}
$$

Definition 4.4. If $P$ is a tableau of shape $\lambda \in \bar{\Gamma}_{0, r}, r \geq n-1$, then the domino word $\pi_{P}$ of $P$ is the signed permutation

$$
\pi_{P}=\left(-C_{m}\right)\left(-C_{m-1}\right) \ldots\left(-C_{1}\right)\left(R_{l}\right)\left(R_{l-1}\right) \ldots\left(R_{1}\right)
$$

where $R_{1}, \ldots, R_{l}$ are the rows having horizontal dominoes and $C_{1}, \ldots, C_{m}$ are the columns having vertical dominoes.

For example,

$$
P=\begin{array}{lllllllllll}
0 & 0 & 0 & 0 & 0 & 1 & 1 & 5 & 5 & 7 & 7 \\
0 & 0 & 0 & 0 & 8 & 8 & & & & & \\
0 & 0 & 0 & & & & & & & & \\
0 & 0 & 9 & & & & & & & & \\
0 & 3 & 9 & & & & & & & & \\
2 & 3 & & & & & & & & & \\
2 & 6 & & & & & & & & & \\
4 & 6 & & & & & & & & &
\end{array}
$$

then the domino word is $\pi_{P}=(-9)(-3)(-6)(-2)(-4)(8)(1)(5)(7)$

Proposition 4.5. If $x, y \in \mathcal{G S P}(n)$ then $x \stackrel{\widetilde{K}}{\sim} y \Longleftrightarrow P(x)=P(y)$ where $P(x), P(y)$ are the standard tableaux of shape $\lambda \in \bar{\Gamma}_{0, r}$, for fixed $r \geq n-1$ obtained using Algorithm BDT in theorem 3.10 .

Proof. The proof is as in Proposition 2.14. We give it for the sake of completion.

We prove as in the case of symmetric group.

$$
\begin{aligned}
x & =\varepsilon_{x_{1}} x_{1} \ldots \varepsilon_{x_{i-1}} x_{i-1} \varepsilon_{x_{i}} x_{i} \varepsilon_{x_{i+1}} x_{i+1} \ldots \varepsilon_{x_{n}} x_{n} \\
y & =\varepsilon_{x_{1}} x_{1} \ldots \varepsilon_{x_{i-1}} x_{i-1} \varepsilon_{x_{i+1}} x_{i+1} \varepsilon_{x_{i}} x_{i} \ldots \varepsilon_{x_{n}} x_{n} .
\end{aligned}
$$


Since all elements inserted before $\varepsilon_{x_{i-2}} x_{i-2}$ are same, it suffices to prove that for any partial tableau inserting $\varepsilon_{x_{i-1}} x_{i-1}, \varepsilon_{x_{i}} x_{i}, \varepsilon_{x_{i+1}} x_{i+1}$ and $\varepsilon_{x_{i-1}} x_{i-1}, \varepsilon_{x_{i+1}} x_{i+1}, \varepsilon_{x_{i}} x_{i}$ in the respective order yield the same tableau. Suppose $\varepsilon_{x_{i-1}}=\varepsilon_{x_{i}}=\varepsilon_{x_{i+1}}=+1$.

If we denote by $I_{x_{i}}(P)$ the tableau rewriting from inserting $\varepsilon_{x_{i}} x_{i}$ in $P$, we have to prove

$$
I_{x_{i-1}} I_{x_{i}} I_{x_{i+1}}(P)=I_{x_{i-1}} I_{x_{i+1}} I_{x_{i}}(P)
$$

The proof is same as in the case of symmetric group, we give for the sake of completion. We prove this claim by induction on the number of rows in $P$. For $P=\delta_{r}$, both sides of equation 4.1 yields the same tableau.

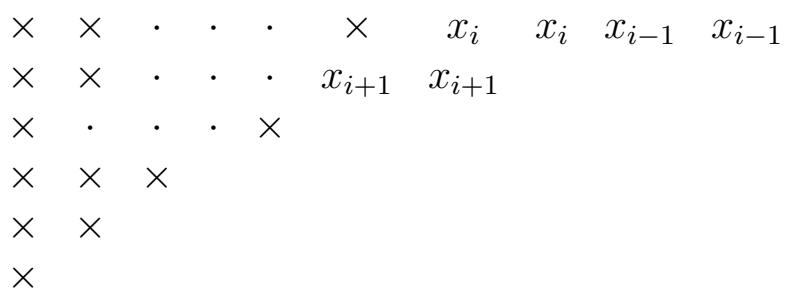

Now assume that $P$ has $r$ rows. Suppose the tablet $x_{i-1} x_{i-1}$ enters in the first row along $k^{\text {th }}$ and $(k+1)^{\text {th }}$ column by replacing \begin{tabular}{|l|l|l|}
\hline$x_{i-1}^{\prime}$ & $x_{i-1}^{\prime}$ & , we examine both sides \\
\hline
\end{tabular} of the equation 4.1. Assume that \begin{tabular}{|l|l|}
\hline$x_{i}$ & $x_{i}$ \\
is inserted next. Since $x_{i}<x_{i-1}, x_{i}$ & $x_{i}$ \\
\hline
\end{tabular} replaces some $x_{i}^{\prime} x_{i}^{\prime}$ from columns $j, j+1$ with $j<k$. Also $x_{i}^{\prime}<x_{i-1}^{\prime}$. This follows from Lemma 2.12 .

Similarly $p_{i+1}>p_{i}$ that \begin{tabular}{|l|l|l|l|}
\hline$x_{i+1}$ & $x_{i+1}$ & replaces some element $x_{i+1}^{\prime}$ & $x_{i+1}^{\prime}$ \\
\hline
\end{tabular} columns $l, l+1$ with $l>k$ and $x_{i+1}^{\prime}>x_{i}^{\prime}$. Considering the right hand side of the equation 4.1, we get that \begin{tabular}{|c|c|c|}
\hline$x_{i+1}$ & $x_{i+1}$
\end{tabular} and $x_{i} x_{i}$ if inserted in this respective order, replace the same elements \begin{tabular}{|l|l|l|}
\hline$x_{i+1}^{\prime}$ & $x_{i+1}^{\prime}$ \\
\hline
\end{tabular} and \begin{tabular}{|l|l|l}
\hline$x_{i}^{\prime}$ & $x_{i}^{\prime}$ \\
\hline
\end{tabular} $j, j+1$.

Therefore the first rows of two tableau obtained are the same. Moreover the rest of tableau is obtained by inserting $x_{i-1}^{\prime}, x_{i}^{\prime}, x_{i+1}^{\prime}$ and $x_{i-1}^{\prime}, x_{i+1}^{\prime}, x_{i}^{\prime}$ in a tableau of a strictly smaller number of rows in this respective order. Since the same order $x_{i}^{\prime}<x_{i-1}^{\prime}<x_{i+1}^{\prime}$ still holds we appeal to induction to asset that the rest of the tableau are also the same.

Suppose $\varepsilon_{x_{i-1}}=\varepsilon_{x_{i}}=\varepsilon_{x_{i+1}}=-1$, then the proof follows by replacing rows by columns in the above case. The argument is the same since positive dominoes insertion along the rows is replaced by negative dominoes insertions along columns.

Similar argument like that of Knuth relation of first kind works for the Knuth relation of third kind. This completes one half of the proof.

We will show that $\pi \stackrel{1}{\sim} \pi_{P}$.

Since Knuth relations are transitive, the converse of the theorem follows.

We induct on $n$. The base case is trivial for $n=1, \pi=\pi_{P}$.

Now assume that $x$ is the last element of $\pi$, that is, $\pi$ is written in one line notation as $\pi=\ldots x$ if $x>0$. 
On $\pi=\pi^{\prime} x$, where $\pi^{\prime}$ is sequence of $n-1$ elements. Therefore, by induction we have $\pi^{\prime} \stackrel{1}{\sim} \pi_{P^{\prime}}$ where $P^{\prime}=P\left(\pi^{\prime}\right)$. Thus it suffices to prove that $\pi_{P^{\prime}} x \stackrel{1}{\sim} \pi_{P}$.

Let $R_{1}, \ldots, R_{l}, C_{1}, \ldots, C_{m}$ be rows and columns of $P^{\prime}$. Assume $R_{1}=p_{1} \cdots p_{k}$. If the domino $x$ enters $P^{\prime}$ in column $j, j+1$, then $p_{1}<\ldots<p_{j-1}<x<p_{j}<\ldots<p_{k}$.

Therefore, we have the following Knuth operation

$$
\begin{aligned}
\pi_{P^{\prime}} x & =\left(-C_{m}\right) \ldots\left(-C_{1}\right)\left(R_{l}\right) \ldots\left(R_{2}\right) p_{1} \ldots p_{k} x \\
& \stackrel{1}{\sim}\left(-C_{m}\right) \ldots\left(-C_{1}\right)\left(R_{l}\right) \ldots\left(R_{2}\right) p_{1} \ldots p_{k-1} x p_{k} \\
& \vdots \\
& \stackrel{1}{\sim}\left(-C_{m}\right) \ldots\left(-C_{1}\right)\left(R_{l}\right) \ldots\left(R_{2}\right) p_{1} \ldots p_{j-1} p_{j} x p_{j+1} \ldots p_{k} \\
& \stackrel{2}{\sim}\left(-C_{m}\right) \ldots\left(-C_{1}\right)\left(R_{l}\right) \ldots\left(R_{2}\right) p_{1} \ldots p_{j} p_{j-1} x p_{j+1} \ldots p_{k} \\
& \vdots \\
& \stackrel{2}{\sim}\left(-C_{m}\right) \ldots\left(-C_{1}\right)\left(R_{l}\right) \ldots\left(R_{2}\right) p_{j} p_{1} \ldots p_{j-1} x p_{j+1} \ldots p_{k}
\end{aligned}
$$

Therefore the Knuth relation generate exactly the first row of $P(\pi)$. Also the element replaced by $x$ from the first row comes at the end of $R_{2}$. The above sequence of operations can be repeated for each row to get the same tableau. The other case is done by replacing row by column. Since the Knuth relation of third kind does not change the relative ordering of elements within the residues of the $P$-tableau remain the same.

Definition 4.6. The generalized signed permutations $x$ and $y$ differ by a dual Knuth relation of first kind, denoted by $x \stackrel{\widetilde{1}^{*}}{\sim} y$ if

$$
\begin{aligned}
x & =\varepsilon_{x_{1}} x_{1} \ldots \varepsilon_{x_{i}} x_{i} \ldots \varepsilon_{x_{i-1}} x_{i-1} \ldots \varepsilon_{x_{i+1}} x_{i+1} \ldots \varepsilon_{x_{n}} x_{n} \text { and } \\
y & =\varepsilon_{x_{1}} x_{1} \ldots \varepsilon_{x_{i+1}} x_{i+1} \ldots \varepsilon_{x_{i-1}} x_{i-1} \ldots \varepsilon_{x_{i}} x_{i} \ldots \varepsilon_{x_{n}} x_{n}
\end{aligned}
$$

such that $x_{i}<x_{i-1}<x_{i+1}$ and $\varepsilon_{x_{i-1}}=\varepsilon_{x_{i}}=\varepsilon_{x_{i+1}}$.

They differ by a Knuth relation of second kind, denoted by $x \stackrel{\widetilde{2}^{*}}{\sim} y$ if

$$
\begin{aligned}
x & =\varepsilon_{x_{1}} x_{1} \ldots \varepsilon_{x_{i-1}} x_{i-1} \ldots \varepsilon_{x_{i+1}} x_{i+1} \ldots \varepsilon_{x_{i}} x_{i} \ldots \varepsilon_{x_{n}} x_{n} \text { and } \\
y & =\varepsilon_{x_{1}} x_{1} \ldots \varepsilon_{x_{i}} x_{i} \ldots \varepsilon_{x_{i+1}} x_{i+1} \ldots \varepsilon_{x_{i-1}} x_{i-1} \ldots \varepsilon_{x_{n}} x_{n}
\end{aligned}
$$

such that $x_{i}<x_{i-1}<x_{i+1}$ and $\varepsilon_{x_{i-1}}=\varepsilon_{x_{i}}=\varepsilon_{x_{i+1}}$.

They differ by a Knuth relation of third kind, denoted by $x \stackrel{\widetilde{3}^{*}}{\sim} y$ if

$$
\begin{aligned}
x & =\varepsilon_{x_{1}} x_{1} \ldots \varepsilon_{x_{i}} x_{i} \ldots \varepsilon_{x_{i+1}} x_{i+1} \ldots \varepsilon_{x_{n}} x_{n} \text { and } \\
y & =\varepsilon_{x_{1}} x_{1} \ldots \varepsilon_{x_{i+1}} x_{i+1} \ldots \varepsilon_{x_{i}} x_{i} \ldots \varepsilon_{x_{n}} x_{n}
\end{aligned}
$$

such that $\varepsilon_{x_{i}}=-\varepsilon_{x_{i+1}}$.

The two permutations are Knuth equivalent, denoted by $x \stackrel{\widetilde{K}^{*}}{\sim} y$ if there is a sequence of permutations such that

$$
x=z_{1} \stackrel{i^{*}}{\sim} z_{2} \stackrel{j^{*}}{\sim} \ldots \stackrel{l^{*}}{\sim} z_{k}=y \text { where } i, j, \ldots, l \in\{\widetilde{1}, \widetilde{2}, \widetilde{3}\} .
$$


Lemma 4.7. If $x, y \in \mathcal{G S P}(n)$ then $x \stackrel{\widetilde{K}}{\sim} y \Longleftrightarrow x^{-1} \stackrel{\widetilde{K}^{*}}{\sim} y^{-1}$.

Proof. The proof follows from the Definitions 4.3 and 4.6.

Proposition 4.8. If $x, y \in \mathcal{G S P}(n)$ then $x \stackrel{\widetilde{K}^{*}}{\sim} y \Longleftrightarrow Q(x)=Q(y)$ where $Q(x), Q(y)$ are the standard tableaux of shape $\lambda \in \bar{\Gamma}_{0, r}$, for fixed $r \geq n-1$ obtained using Algorithm BDT in theorem 3.10.

Proof. The proof is as in the case of symmetric group.

$$
\begin{aligned}
x \stackrel{\widetilde{K}^{*}}{\sim} y & \Longleftrightarrow x^{-1} \stackrel{\widetilde{K}}{\sim} y^{-1} & & (\text { Lemma } 4.7) \\
& \Longleftrightarrow P\left(x^{-1}\right)=P\left(y^{-1}\right) & & \text { (Proposition } 4.5) \\
& \Longleftrightarrow Q(x)=Q(y) & & \text { (Proposition } 4.2) .
\end{aligned}
$$

Definition 4.9. Let $d, d^{\prime} \in \bar{V}_{n}$. Then $d$ and $d^{\prime}$ are Knuth equivalent, denoted by $d \stackrel{K}{\sim} d^{\prime}$ if the following condition holds.

1. $d_{2}=d_{2}^{\prime}$.

2. $w \stackrel{\widetilde{K}}{\sim} w^{\prime}$ where $w, w^{\prime} \in \mathcal{G S P}(t), t$ is the number of vertical edges in $d$.

Proposition 4.10. Let $d, d^{\prime} \in \bar{V}_{n}$. Then $d \stackrel{K}{\sim} d^{\prime} \Longleftrightarrow\left(P_{1}(d), P_{2}(d)\right)=\left(P_{1}\left(d^{\prime}\right), P_{2}\left(d^{\prime}\right)\right)$ where $P_{1}(d), P_{1}\left(d^{\prime}\right)$ are the column standard block tableaux of shape $\lambda_{1}=\left(2^{2 f}\right)$ and $P_{2}(d), P_{2}\left(d^{\prime}\right)$ are the standard tableaux of shape $\lambda_{2} \in \bar{\Gamma}_{f, r}$, for fixed $r \geq n-1$ and $0 \leq f \leq\left[\frac{n}{2}\right]$.

Proof. The proof follows from the Definition 4.9 and by the Proposition 4.5

Corollary 4.11. Let $d$ and $d^{\prime}$ be any two Brauer diagrams in $\bar{V}_{n}$ having only positive edges. Then $d \stackrel{K}{\sim} d^{\prime} \Longleftrightarrow\left(P_{1}(d), P_{2}(d)\right)=\left(P_{1}\left(d^{\prime}\right), P_{2}\left(d^{\prime}\right)\right)$ where $P_{1}(d), P_{1}\left(d^{\prime}\right)$ are the column standard tableaux of shape $\lambda_{1}=\left(2^{f}\right)$ and $P_{2}(d), P_{2}\left(d^{\prime}\right)$ are the standard tableaux of shape $\lambda_{2}, \lambda_{2} \in \Gamma_{f}$ where $\Gamma_{f}=\{\lambda \mid \lambda \vdash n-2 f\}$ and $0 \leq f \leq\left[\frac{n}{2}\right]$.

Proof. The proof follows by the above proposition and the corollary 3.13.

Definition 4.12. Let $d, d^{\prime} \in \bar{V}_{n}$. Then $d$ and $d^{\prime}$ are dual Knuth equivalent, denoted by $d \stackrel{K^{*}}{\sim} d^{\prime}$ if the following condition holds.

1. $d_{1}=d_{1}^{\prime}$.

2. $w \stackrel{\widetilde{K}^{*}}{\sim} w^{\prime}$ where $w, w^{\prime} \in \mathcal{G S P}(t), t$ is the number of vertical edges in $d$.

Proposition 4.13. Let $d, d^{\prime} \in \bar{V}_{n}$. Then $d \stackrel{K^{*}}{\sim} d^{\prime} \Longleftrightarrow\left(Q_{1}(d), Q_{2}(d)\right)=\left(Q_{1}\left(d^{\prime}\right), Q_{2}\left(d^{\prime}\right)\right)$ where $Q_{1}(d), Q_{1}\left(d^{\prime}\right)$ are the column standard block tableaux of shape $\lambda_{1}=\left(2^{2 f}\right)$ and $Q_{2}(d), Q_{2}\left(d^{\prime}\right)$ are the standard tableaux of shape $\lambda_{2} \in \bar{\Gamma}_{f, r}$, for fixed $r \geq n-1$ and $0 \leq f \leq\left[\frac{n}{2}\right]$. 
Proof. The proof follows from the Definition 4.12 and by the Proposition 4.8

Corollary 4.14. Let $d$ and $d^{\prime}$ be any two Brauer diagrams $\bar{V}_{n}$ having only positive edges. Then $d \stackrel{K^{*}}{\sim} d^{\prime} \Longleftrightarrow\left(Q_{1}(d), Q_{2}(d)\right)=\left(Q_{1}\left(d^{\prime}\right), Q_{2}\left(d^{\prime}\right)\right)$ where $Q_{1}(d), Q_{1}\left(d^{\prime}\right)$ are the column standard tableaux of shape $\lambda_{1}=\left(2^{f}\right)$ and $Q_{2}(d), Q_{2}\left(d^{\prime}\right)$ are the standard tableaux of shape $\lambda_{2}, \lambda_{2} \in \Gamma_{f}$ where $\Gamma_{f}=\{\lambda \mid \lambda \vdash n-2 f\}$ and $0 \leq f \leq\left[\frac{n}{2}\right]$.

Proof. The proof follows by the above proposition and the corollary 3.13 .

\subsection{The Determinantal Formula}

Set $\frac{1}{r !}=0$ if $r<0$.

Lemma 4.15. $\left|\begin{array}{ccccc}\overline{2^{0} 0 !} & \overline{2^{1} 1 !} & \overline{2^{2} 2 !} & \cdots & \overline{2^{f-1}(f-1) !} \\ 0 & \frac{1}{2^{0} 0 !} & \frac{1}{2^{1} 1 !} & \cdots & \frac{1}{2^{f-2}(f-2) !} \\ \vdots & \vdots & \vdots & \vdots & \vdots \\ 0 & 0 & 0 & \cdots & \frac{1}{2^{1} 1 !}\end{array}\right|=\frac{1}{2^{f} f !}$

$$
\begin{aligned}
& \text { Lemma 4.15. }\left|\begin{array}{ccccc}
\frac{1}{2^{1} 1 !} & \frac{1}{2^{2} 2 !} & \frac{1}{2^{3} 3 !} & \cdots & \frac{1}{2^{f} f !} \\
\frac{1}{2^{0} 0 !} & \frac{1}{2^{1} 1 !} & \frac{1}{2^{2} 2 !} & \cdots & \frac{1}{2^{f-1}(f-1) !} \\
0 & \frac{1}{2^{0} 0 !} & \frac{1}{2^{1} 1 !} & \cdots & \frac{1}{2^{f-2}(f-2) !} \\
\vdots & \vdots & \vdots & \vdots & \\
0 & 0 & 0 & \cdots & \frac{1}{2^{1} 1 !}
\end{array}\right|=\frac{1}{2^{f} f !} \\
& \text { Proof. Let } \left.A_{f}=\mid \begin{array}{cccccc}
\frac{1}{2^{1} 1 !} & \frac{1}{2^{2} 2 !} & \frac{1}{2^{3} 3 !} & \cdots & \frac{1}{2^{f} f !} \\
\frac{1}{2^{0} 0 !} & \frac{1}{2^{1} 1 !} & \frac{1}{2^{2} 2 !} & \cdots & \frac{1}{2^{f-1}(f-1) !} \\
0 & \frac{1}{2^{0} 0 !} & \frac{1}{2^{1} 1 !} & \cdots & \frac{1}{2^{f-2}(f-2) !} \\
\vdots & \vdots & \vdots & \vdots & \vdots \\
0 & 0 & 0 & \cdots & \frac{1}{2^{1} 1 !}
\end{array}\right) .
\end{aligned}
$$

We prove the lemma by

induction.

$$
\left|A_{f}\right|=\left|\begin{array}{ccccc}
\frac{1}{2^{1} 1 !} & \frac{1}{2^{2} 2 !} & \frac{1}{2^{3} 3 !} & \cdots & \frac{1}{2^{f} f !} \\
\frac{1}{2^{0} 0 !} & \frac{1}{2^{1} 1 !} & \frac{1}{2^{2} 2 !} & \cdots & \frac{1}{2^{f-1}(f-1) !} \\
0 & \frac{1}{2^{0} 0 !} & \frac{1}{2^{1} 1 !} & \cdots & \frac{1}{2^{f-2}(f-2) !} \\
\vdots & \vdots & \vdots & \vdots & \vdots \\
0 & 0 & 0 & \cdots & \frac{1}{2^{1} 1 !}
\end{array}\right|
$$




$$
\begin{aligned}
= & \frac{1}{2^{1} 1 !}\left|A_{f-1}\right|-\frac{1}{2^{2} 2 !}\left|A_{f-2}\right|+\cdots+\frac{(-1)^{f}}{2^{f-1}(f-1) !}\left|A_{1}\right|+\frac{(-1)^{f+1}}{2^{f} f !} \\
= & \frac{1}{2^{1} 1 !} \frac{1}{2^{f-1}(f-1) !}-\frac{1}{2^{2} 2 !} \frac{1}{2^{f-2}(f-2) !}+\cdots+\frac{(-1)^{f}}{2^{f-1}(f-1) !} \frac{1}{2^{1} 1 !} \\
& +\frac{(-1)^{f+1}}{2^{f} f !}(\text { by induction }) \\
= & \frac{1}{2^{f} 1 !(f-1) !}-\frac{1}{2^{f} 2 !(f-2) !}+\cdots+\frac{(-1)^{f}}{2^{f}(f-1) ! 1 !}+\frac{(-1)^{f+1}}{2^{f} f !} \\
= & \frac{1}{2^{f}}\left[\frac{1}{1 !(f-1) !}-\frac{1}{2 !(f-2) !}+\cdots+\frac{(-1)^{f}}{(f-1) ! 1 !}+\frac{(-1)^{f+1}}{f !}\right] \\
= & \frac{1}{2^{f} f !}
\end{aligned}
$$

Proposition 4.16. If $\lambda=\left(2^{2 f}\right)$ then

$$
g(\lambda)=2^{f}(2 f) ! !=2^{f}(2 f) !\left|\frac{1}{2^{\left(\lambda_{i}-i+j-1\right)}\left(\lambda_{i}-i+j-1\right) !}\right|_{f \times f}
$$

where the determinant is $f \times f$ and $g(\lambda)$ is the number of column standard block tableau of shape $\lambda=\left(2^{2 f}\right)$.

Proof. Let $1,2, \ldots, 2 f$ be the $2 f$ digits. Split the digits into $f$ pairs $\left(i_{l}, j_{l}\right), l=1,2, \ldots, f$ by choosing the pairs such that for $l, m=1,2, \ldots, f$ and $r=1,2, \ldots, f-1$,

$$
i_{r}<i_{r+1}, \quad i_{l}<j_{l}, \quad i_{l} \neq j_{m} \text { for } l \neq m, j_{l} \neq j_{m} \text { for } l \neq m .
$$

Number of such choices is $(2 f) ! !$ and the number of standard bidomino of shape $\left(2^{2}\right)$ is 2. i.e. The number of row standard bi-dominotableau of shape $\lambda=\left(2^{2 f}\right)$ is $2^{f}(2 f) !$ !.

It suffices to prove $(2 f) ! !=(2 f) !\left|\frac{1}{2^{\left(\lambda_{i}-i+j-1\right)}\left(\lambda_{i}-i+j-1\right) !}\right|_{f \times f}$.

i.e. $\left|\frac{1}{2^{\left(\lambda_{i}-i+j-1\right)}\left(\lambda_{i}-i+j-1\right) !}\right|_{f \times f}=\frac{(2 f) ! !}{(2 f) !}=\frac{1}{2^{f} f !}$. 
Since $\lambda_{i}=2, \forall 1 \leq i \leq f$,

$$
\begin{aligned}
& \left|\frac{1}{2^{\left(\lambda_{i}-i+j-1\right)}\left(\lambda_{i}-i+j-1\right) !}\right|_{f \times f}=\left|\frac{1}{2^{(j-i+1)}(j-i+1) !}\right|_{f \times f} \\
& =\left|\begin{array}{ccccc}
\frac{1}{2^{1} 1 !} & \frac{1}{2^{2} 2 !} & \frac{1}{2^{3} 3 !} & \cdots & \frac{1}{2^{f} f !} \\
\frac{1}{2^{0} 0 !} & \frac{1}{2^{1} 1 !} & \frac{1}{2^{2} 2 !} & \cdots & \frac{1}{2^{f-1}(f-1) !} \\
0 & \frac{1}{2^{0} 0 !} & \frac{1}{2^{1} 1 !} & \cdots & \frac{1}{2^{f-2}(f-2) !} \\
\vdots & \vdots & \vdots & \vdots & \vdots \\
0 & 0 & 0 & \cdots & \frac{1}{2^{1} 1 !}
\end{array}\right| \\
& =\frac{1}{2^{f} f !} \text { (by Lemma 4.15). }
\end{aligned}
$$

Theorem 4.17. (Determinantal Formula) If $\rho=(\lambda, \mu)$ with $\lambda=\left(2^{2 f}\right), \mu \in \bar{\Gamma}_{f, r}$, for fixed $r \geq 0$ where $0 \leq f \leq\left[\frac{n}{2}\right]$ then

$$
\begin{aligned}
h^{\rho}= & 2^{f} n !\left|\frac{1}{2^{\left(\lambda_{i}-i+j-1\right)}\left(\lambda_{i}-i+j-1\right) !}\right|_{f \times f}\left|\frac{1}{\left(\mu_{i}^{(1)}-i+j\right) !}\right|_{l \times l} \\
& \left|\frac{1}{\left(\mu_{i}^{(2)}-i+j\right) !}\right|_{n-2 f-l \times n-2 f-l}
\end{aligned}
$$

where $\mu^{(1)} \vdash l$ and $\mu^{(2)} \vdash n-2 f-l$ as in Definition 2.15 and $h^{\rho}$ is the standard $\rho$-bi-domino tableau.

Proof. Let $\mu \in \bar{\Gamma}_{f, r}$, for fixed $r \geq 0$ where $0 \leq f \leq\left[\frac{n}{2}\right]$ then by Definition 2.15, $\mu=\left(\mu^{(1)}, \mu^{(2)}\right)$ where $\mu^{(1)} \vdash l$ and $\mu^{(2)} \vdash n-2 f-l$.

$$
\begin{aligned}
h^{\rho}= & \left(\begin{array}{c}
n \\
2 f
\end{array}\right) g(\lambda) f^{\mu} \\
= & \left(\begin{array}{c}
n \\
2 f
\end{array}\right) 2^{f}(2 f) !\left|\frac{1}{2^{\left(\lambda_{i}-i+j-1\right)}\left(\lambda_{i}-i+j-1\right) !}\right|_{f \times f}(n-2 f) !\left|\frac{1}{\left(\mu_{i}^{(1)}-i+j\right) !}\right|_{l \times l} \\
= & 2^{f} n !\left|\frac{1}{\left.2^{(2)}-i+j\right) !}\right|_{n-2 f-l \times n-2 f-l} \text { (by Proposition 2.16 and Proposition 4.16) } \\
& \left|\frac{1}{\left(\mu_{i}^{(2)}-i+j\right) !}\right|_{n-2 f-l \times n-2 f-l}
\end{aligned}
$$


Corollary 4.18. If $\rho=(\lambda, \mu)$ with $\lambda=\left(2^{f}\right), \mu \in \Gamma_{f}$ where $\Gamma_{f}=\{\lambda \mid \lambda \vdash n-2 f\}$ and $0 \leq f \leq\left[\frac{n}{2}\right]$ then

$$
h^{\rho}=n !\left|\frac{1}{2^{\left(\lambda_{i}-i+j-1\right)}\left(\lambda_{i}-i+j-1\right) !}\right|_{f \times f}\left|\frac{1}{\left(\mu_{i}-i+j\right) !}\right|_{n-2 f \times n-2 f}
$$

where $h^{\rho}$ is the standard $\rho$-bi tableau.

Proof. The proof follows by the above theorem and the corollary 3.13.

\section{Acknowledgement}

We would like to express our sincere thanks to the referee for his suggestions and comments for the improvement of the paper. We also thank the referee for the Remark 3.20.

\section{References}

[A] A. Ram, Characters of Brauer's centralizer algebras, Pac.J.Math. 169 (1) (1995), 173-200.

[BI] C. Bonnafé and L. Iancu, Left cells in type $B_{n}$ with unequal parameters, preprint, 2003; available at arXiv: math.RT/0302037.

[Br] R. Brauer, On algebras which are connected with the semisimple continuous groups, Ann.Math. 38 (1937), 854-872.

[E] John Enyang, Cellular bases for the Brauer and Birman-Murakami-Wenzl algebras, J.Algebra 281 (2004) 413-449.

[DS] S. Dulucq and B. Sagan, La correspondence de Robinson-Schensted pour les tableaux oscillants gauches, Discrete Math, 139 (1995), 129-142.

[HL] T. Halverson and T. Lewandowski, RSK insertion for set partitions and diagram algebras, Electronic J.Combinatorics, 11(2) R24, 2004-2005.

[JK] G. James and A. Kerber, The Representation theory of the symmetric group, Encyclopedia of mathematics and its applications, Addison Wesley publishing company 1981.

[Knu] D. E. Knuth, Permutaions, matrices and generalized Young tableaux, Pacific J. Math. 34 (1970), 709-727.

[PK] M. Parvathi and M. Kamaraj, Signed Brauer Algebras, Comm. in Algebra, 26(3) (1998), 839-855.

[PST] M. Parvathi, B. Sivakumar and A. Tamilselvi, $R-S$ correspondence for the hyperoctahedral group of type $B_{n}-A$ different approach, J. Algebra and Discrete Mathematics, 1(2007) 87-108.

[R] G. de B. Robinson, it Representation theory of symmetric group, Edinburg University press, 1961. 
[Ro1] T. Roby, Applications and Extensions of Fomin's Generalization of the RobinsonSchensted Correspondence to Differential Posets, Ph.D. Thesis, M.I.T., 1991.

[Ro2] T. Roby, The connection between the Robinson-Schensted correspondence for skew oscillating tableaux and graded graphs, Discrete Math, 139 (1995), 481-485.

[S] Bruce E. Sagan, The Symmetric Group, second edition, Springer 1991.

[Su] S. Sundaram, On the combinatorics of representations of $S p(2 n, \mathbb{C})$, Ph.D. Thesis, M.I.T., 1986. 